\title{
Global well-posedness of compressible Navier-Stokes equations for some classes of large initial data
}

\author{
Chao Wang ${ }^{\dagger}$, Wei Wang ${ }^{\ddagger}$ and Zhifei Zhang ${ }^{\ddagger}$ \\ $\dagger$ Academy of Mathematics \& Systems Science, CAS, Beijing 100190, China \\ E-mail: wangchao@amss.ac.cn \\ $\ddagger$ LMAM and School of Mathematical Science, Peking University, Beijing 100871, China \\ E-mail: wangw07@pku.edu.cn, zfzhang@math.pku.edu.cn
}

November 13, 2011

\begin{abstract}
We prove the global well-posedness of three dimensional compressible Navier-Stokes equations for some classes of large initial data, which is of large oscillation for the density and large energy for the velocity. The structure of the system (especially, the effective viscous flux) is fully exploited.
\end{abstract}

\section{Introduction}

We consider the three dimensional compressible Navier-Stokes equations

$$
\left\{\begin{array}{l}
\partial_{t} \rho+\operatorname{div}(\rho u)=0 \\
\partial_{t}(\rho u)+\operatorname{div}(\rho u \otimes u)-\mu \Delta u-\lambda \nabla \operatorname{div} u+\nabla P(\rho)=0 \\
\left.(\rho, u)\right|_{t=0}=\left(\rho_{0}, u_{0}\right) .
\end{array}\right.
$$

Here $\rho(t, x)$ and $u(t, x)$ denote the density and velocity of the fluid respectively. The Lamé coefficients $\mu$ and $\lambda$ satisfy

$$
\mu>0 \text { and } \lambda+\mu>0
$$

which ensure that the operator $\mu \Delta u+\lambda \nabla \operatorname{div} u$ is elliptic. The pressure $P$ is a smooth function of $\rho$ and satisfies

$$
\begin{gathered}
P(0)=0, \quad P^{\prime}(\bar{\rho})>0 \\
(\rho-\bar{\rho})(P(\rho)-P(\bar{\rho}))>0 \quad \text { for } \quad \rho \neq \bar{\rho}, \rho \in\left[0,2 c_{0}^{-1}\right],
\end{gathered}
$$

where $0<c_{0}<\bar{\rho}<c_{0}^{-1}$ are fixed numbers.

The local existence and uniqueness of smooth solution of the system (1.1) was proved by Nash [31] for smooth initial data without vacuum. In a seminal paper [30, Matsumura and Nishida proved the global existence and uniqueness of smooth solution for smooth initial data close to equilibrium. In general, whether smooth solution blows up in finite time is an 
open problem. However, Xin [34 proved that smooth solution will blow up in finite time if the initial density has compact support. Recently, Sun, Wang and Zhang [33] showed that smooth solution does not blow up if the concentration of the density does not occur.

The global existence of weak solution was proved by Hoff [22, 25] for the discontinuous initial data with small energy. For the large initial data, the global existence of weak solution was proved by Lions [29] for the isentropic Navier-Stokes equation, i.e. $P=A \rho^{\gamma}$ for $\gamma \geq \frac{9}{5}$. Feireisl, Novotný and Petzeltová [20] improved Lions's result to $\gamma>\frac{3}{2}$. For the spherically symmetric or axisymmetric initial data, Jiang and Zhang [27, 28] proved the global existence of weak solution for any $\gamma>1$. However, the question of the regularity and uniqueness of weak solution is completely open even in the case of two dimensional space.

Motivated by Fujita and Kato's result for the incompressible Navier-Stokes equations [21, Danchin in a series of papers [15, 16, 17, 19] proved the local well-posedness of (1.1) for the initial data $\left(\rho_{0}-\bar{\rho}, u_{0}\right)$ belonging to the critical Besov spaces $\dot{B}_{p, 1}^{\frac{3}{p}} \times \dot{B}_{p, 1}^{\frac{3}{p}-1}$ for $p<6$, and global existence for the initial data close to equilibrium in $\dot{B}_{2,1}^{\frac{3}{2}} \times \dot{B}_{2,1}^{\frac{1}{2}}$. This seems the largest space in which the system (1.1) is well-posed. Indeed, Chen, Miao and Zhang [14] proved the ill-posedness of (1.1) in $\dot{B}_{p, 1}^{\frac{3}{p}} \times \dot{B}_{p, 1}^{\frac{3}{p}-1}$ for $p>6$.

Cannone, Meyer and Planchon [3, 4, proved the global existence of the solution for the incompressible Navier-Stokes equations in the Besov space of negative regularity index $\dot{B}_{p, \infty}^{\frac{3}{p}-1}$ for $p>3$, see also [1] for a new proof based on the smoothing effect of the heat equation. A very interesting point of this result is that it allows to construct global solution for the highly oscillating initial velocity that may have a large norm in $\dot{H}^{\frac{1}{2}}$ or $L^{3}$. A typical example is

$$
u_{0}(x)=\sin \left(\frac{x_{3}}{\varepsilon}\right)\left(-\partial_{2} \phi(x), \partial_{1} \phi(x), 0\right)
$$

where $\phi \in \mathcal{S}\left(\mathbf{R}^{3}\right)$ and $\varepsilon>0$ is small enough. Later, Chemin, Gallagher, and Paicu [7, 9] find more classes of initial data with large energy generating the global solution. And in the proof of [9], the special structure of the equation is used. We refer to [11, [8, 10, 32, 36] and references therein for more relevant results.

It is highly non trivial to generalize Cannone-Meyer-Planchon's result to (1.1), since it is a hyperbolic-parabolic coupled system. Very recently, important progress has been made by Chen, Miao and Zhang [13] and Charve and Danchin [5], where they construct the global solution of (1.1) for the highly oscillating initial veloicty by proving the global well-posedness of the system (1.1) in the critical Besov space with $p>3$.

However, for all the above global existence results, the initial density is required to be close to a positive constant in $L^{\infty}$ norm, which precludes the oscillation of the density at any point. Recently, Zhang [35] proved the global existence and uniqueness of (1.1) for the initial density $\rho_{0}$ close to a positive constant in $L^{2}$ norm and $u_{0}$ small in $L^{p}$ norm for $p>3$, which allows the density to have large oscillation on a set of small measure. Similar result has also been obtained by Huang, Li and Xin [26] for the initial data with vacuum, but a compatibility condition is imposed on the initial data.

The proof of [35] and [26] is based on Hoff's energy method, where the structure of the system is fully exploited, especially the effective viscous flux plays an important role. While, the proof of [13] and [5] is based on the analysis for the linearized system, where Harmonic analysis tools (especially Littlewood-Paley theory) are exploited, but the structure of the system is not exploited. 
In this paper, we combine two methods to find a new class of large initial data generating the global solution of the system (1.1). This class of initial data may have large oscillation for the density and large energy for the velocity. More precisely, we prove

Theorem 1.1 Assume that the initial data $\left(\rho_{0}, u_{0}\right)$ satisfies

$$
\rho_{0}-\bar{\rho} \in H^{s} \cap \dot{B}_{p, 1}^{\frac{3}{p}}, \quad c_{0} \leq \rho_{0} \leq c_{0}^{-1}, \quad u_{0} \in H^{s-1} \cap \dot{B}_{p, 1}^{\frac{3}{p}-1}
$$

for some $p \in(3,6)$ and $s \geq 3$. There exist two constants $c_{1}=c_{1}\left(\lambda, \mu, c_{0}, \bar{\rho}\right)$ and

$$
c_{2}=\frac{1}{C\left(1+\left\|\rho_{0}-\bar{\rho}\right\|_{\dot{B}_{p, 1}^{\frac{3}{p}} \cap H^{s}}+\left\|u_{0}\right\|_{2}\right)^{5}}, \quad C=C\left(\lambda, \mu, c_{0}, \bar{\rho}\right)
$$

such that if

$$
\left\|\rho_{0}-\bar{\rho}\right\|_{L^{2}} \leq c_{1}, \quad\left\|u_{0}\right\|_{\dot{H}^{-\delta}}+\left\|u_{0}\right\|_{\dot{B}_{p, 1}^{\frac{3}{p}-1}} \leq c_{2}
$$

for some $\delta \in\left(1-\frac{3}{p}, \frac{3}{p}\right)$, then there exits a unique global solution $(\rho, u)$ to the compressible Navier-Stokes system (1.1) satisfying

$$
\begin{gathered}
\rho \geq \frac{c_{0}}{4}, \quad \rho-\bar{\rho} \in C\left([0,+\infty) ; H^{s}\right), \\
u \in C\left([0,+\infty) ; H^{s-1}\right) \cap L^{2}\left(0, T ; H^{s}\right) \quad \text { for any } \quad T>0 .
\end{gathered}
$$

Remark 1.2 Since the constant $c_{1}$ is independent of $\rho_{0}$, the condition $\left\|\rho_{0}-\bar{\rho}\right\|_{L^{2}} \leq c_{1}$ allows the initial density to have large oscillation on the set with small measure. Given $\rho_{0}$, if we take the the highly oscillating initial velocity, for example,

$$
u_{0}(x)=\sin \left(\frac{x}{\varepsilon}\right) \varphi(x)
$$

for any $\varphi \in \mathcal{S}\left(\mathbf{R}^{3}\right)$, then for $p>3$ and $\varepsilon$ small enough,

$$
\left\|u_{0}\right\|_{\dot{H}^{-\delta}}+\left\|u_{0}\right\|_{\dot{B}_{p, 1}^{\frac{3}{p}-1}} \leq C \varepsilon^{\min \left(\delta, 1-\frac{3}{p}\right)} \leq c_{2} .
$$

Interestingly, $u_{0}$ has a large norm in $H^{s}$ for $s \geq 0$, hence the large energy.

Remark 1.3 Whether similar result can be generalized to the case of two dimensional space remains unknown. However, it seems impossible to generalize this result to the full NavierStokes equations, see [14].

The main steps of the proof are as follows. First of all, we construct a local solution of (1.1) for the initial data $\left(\rho_{0}-\bar{\rho}, u_{0}\right)$ belonging to the critical Besov space $\dot{B}_{p, 1}^{\frac{3}{p}} \times \dot{B}_{p, 1}^{\frac{3}{p}-1}$. To deal with the case of physical non vacuum (i.e., $\rho_{0} \geq c_{0}>0$ ), we need to use the weighted Besov space introduced by Chen, Miao and Zhang [12]. Using the smoothing effect of the momentum equation, this solution can propagate the smallness of the initial velocity in Sobolev space of negative regularity index to that of the velocity at some time $t_{0}>0$ in Sobolev space of positive regularity index. Then Hoff's energy method can be applied to prove that the density is bounded below and above after $t>t_{0}$. Finally, we prove a continuation criterion of smooth solution, which ensures that the local solution can be extended to a global solution. 


\section{Littlewood-Paley analysis}

\subsection{Littlewood-Paley decomposition}

Let us introduce the Littlewood-Paley decomposition. Choose a radial function $\varphi \in \mathcal{S}\left(\mathbf{R}^{3}\right)$ supported in $\mathcal{C}=\left\{\xi \in \mathbf{R}^{3}, \frac{3}{4} \leq|\xi| \leq \frac{8}{3}\right\}$ such that

$$
\sum_{j \in \mathbf{Z}} \varphi\left(2^{-j} \xi\right)=1 \quad \text { for all } \xi \neq 0
$$

The frequency localization operator $\Delta_{j}$ and $S_{j}$ are defined by

$$
\Delta_{j} f=\varphi\left(2^{-j} D\right) f, \quad S_{j} f=\sum_{k \leq j-1} \Delta_{k} f \quad \text { for } \quad j \in \mathbf{Z} .
$$

With our choice of $\varphi$, one can easily verify that

$$
\begin{aligned}
& \Delta_{j} \Delta_{k} f=0 \quad \text { if } \quad|j-k| \geq 2 \text { and } \\
& \Delta_{j}\left(S_{k-1} f \Delta_{k} f\right)=0 \text { if }|j-k| \geq 5 .
\end{aligned}
$$

Next we recall Bony's decomposition from [2]:

$$
u v=T_{u} v+T_{v} u+R(u, v)
$$

with

$$
T_{u} v=\sum_{j \in \mathbf{Z}} S_{j-1} u \Delta_{j} v, \quad R(u, v)=\sum_{j \in \mathbf{Z}} \Delta_{j} u \widetilde{\Delta}_{j} v, \quad \widetilde{\Delta}_{j} v=\sum_{\left|j^{\prime}-j\right| \leq 1} \Delta_{j^{\prime}} v .
$$

The following Bernstein lemma will be frequently used(see [1]).

Lemma 2.1 Let $1 \leq p \leq q \leq+\infty$. Assume that $f \in L^{p}\left(\mathbf{R}^{3}\right)$, then for any $\gamma \in(\mathbf{N} \cup\{0\})^{3}$, there exist constants $C_{1}, C_{2}$ independent of $f, j$ such that

$$
\begin{aligned}
& \operatorname{supp} \hat{f} \subseteq\left\{|\xi| \leq A_{0} 2^{j}\right\} \Rightarrow\left\|\partial^{\gamma} f\right\|_{q} \leq C_{1} 2^{j|\gamma|+3 j\left(\frac{1}{p}-\frac{1}{q}\right)}\|f\|_{p}, \\
& \operatorname{supp} \hat{f} \subseteq\left\{A_{1} 2^{j} \leq|\xi| \leq A_{2} 2^{j}\right\} \Rightarrow\|f\|_{p} \leq C_{2} 2^{-j|\gamma|} \sup _{|\beta|=|\gamma|}\left\|\partial^{\beta} f\right\|_{p} .
\end{aligned}
$$

\subsection{The functional spaces}

We denote the space $\mathcal{Z}^{\prime}\left(\mathbf{R}^{3}\right)$ by the dual space of $\mathcal{Z}\left(\mathbf{R}^{3}\right)=\left\{f \in \mathcal{S}\left(\mathbf{R}^{3}\right) ; D^{\alpha} \hat{f}(0)=0 ; \forall \alpha \in\right.$ $\mathbf{N}^{3}$ multi-index $\}$, which can be identified by the quotient space of $\mathcal{S}^{\prime}\left(\mathbf{R}^{3}\right) / \mathcal{P}$ with the polynomials space $\mathcal{P}$.

Definition 2.2 Let $s \in \mathbf{R}, 1 \leq p, r \leq+\infty$. The homogeneous Besov space $\dot{B}_{p, r}^{s}$ is defined by

$$
\dot{B}_{p, r}^{s}=\left\{f \in \mathcal{Z}^{\prime}\left(\mathbf{R}^{3}\right):\|f\|_{\dot{B}_{p, r}^{s}}<+\infty\right\},
$$

where

$$
\|f\|_{\dot{B}_{p, r}^{s}} \stackrel{\text { def }}{=}\left\|2^{k s}\right\| \Delta_{k} f(t)\left\|_{p}\right\|_{\ell^{r}} .
$$

In particular, $\dot{B}_{2,2}^{s}=\dot{H}^{s}, \dot{H}^{s}$ is the homogeneous Sobolev space. 
Let $\left\{e_{k}(t)\right\}_{k \in \mathbf{Z}}$ be a sequence defined in $[0,+\infty)$ satisfying the following conditions

$$
e_{k}(t) \in[0,1], \quad e_{k}(t) \leq e_{k^{\prime}}(t) \quad \text { if } \quad k \leq k^{\prime} \quad \text { and } \quad e_{k}(t) \sim e_{k^{\prime}}(t) \quad \text { if } \quad k \sim k^{\prime},
$$

where $k \sim k^{\prime}$ means that there exists a constant $N_{0}$ such that $\left|k-k^{\prime}\right| \leq N_{0}$. The weight function $\left\{\omega_{k}(t)\right\}_{k \in \mathbf{Z}}$ is defined by

$$
\omega_{k}(t)=\sum_{\ell \geq k} 2^{k-\ell} e_{\ell}(t), \quad k \in \mathbf{Z}
$$

It is easy to verify that for any $k \in \mathbf{Z}$,

$$
\begin{aligned}
& \omega_{k}(t) \leq 2, \quad e_{k}(t) \leq \omega_{k}(t), \\
& \omega_{k}(t) \leq 2^{k-k^{\prime}} \omega_{k^{\prime}}(t) \quad \text { if } k \geq k^{\prime}, \quad \omega_{k}(t) \leq 3 \omega_{k^{\prime}}(t) \quad \text { if } k \leq k^{\prime}, \\
& \omega_{k}(t) \sim \omega_{k^{\prime}}(t) \quad \text { if } k \sim k^{\prime} .
\end{aligned}
$$

The following weighted Besov space is introduced in [12].

Definition 2.3 Let $s \in \mathbf{R}, 1 \leq p, r \leq+\infty, 0<T<+\infty$. The weighted Besov space $\dot{B}_{p, r}^{s}(\omega)$ is defined by

$$
\dot{B}_{p, r}^{s}(\omega)=\left\{f \in \mathcal{Z}^{\prime}\left(\mathbf{R}^{N}\right):\|f\|_{\dot{B}_{p, r}^{s}(\omega)}<+\infty\right\},
$$

where

$$
\|f\|_{\dot{B}_{p, r}^{s}(\omega)} \stackrel{\text { def }}{=}\left\|2^{k s} \omega_{k}(T)\right\| \Delta_{k} f\left\|_{p}\right\|_{\ell^{r}}
$$

Obviously, $\dot{B}_{p, r}^{s} \subset \dot{B}_{p, r}^{s}(\omega)$ and

$$
\|f\|_{\dot{B}_{p, r}^{s}(\omega)} \leq 2\|f\|_{\dot{B}_{p, r}^{s}}
$$

We also need to use Chemin-Lerner type Besov spaces introduced in [6].

Definition 2.4 Let $s \in \mathbf{R}, 1 \leq p, q, r \leq+\infty, 0<T \leq+\infty$. The functional space $\widetilde{L}_{T}^{q}\left(\dot{B}_{p, r}^{s}\right)$ is defined as the set of all the distributions $f$ satisfying

$$
\|f\|_{\widetilde{L}_{T}^{q}\left(\dot{B}_{p, r}^{s}\right)} \stackrel{\text { def }}{=}\left\|2^{k s}\right\| \Delta_{k} f(t)\left\|_{L^{q}\left(0, T ; L^{p}\right)}\right\|_{\ell^{r}}<+\infty
$$

The weighted functional space $\widetilde{L}_{T}^{q}\left(\dot{B}_{p, 1}^{s}(\omega)\right)$ is defined similarly, whose norm is given by

$$
\|f\|_{\widetilde{L}_{T}^{q}\left(\dot{B}_{p, 1}^{s}(\omega)\right)} \stackrel{\text { def }}{=}\left\|2^{k s} \omega_{k}(T)\right\| \Delta_{k} f(t)\left\|_{L^{q}\left(0, T ; L^{p}\right)}\right\|_{\ell^{r}} .
$$

\subsection{Nonlinear estimates in Besov spaces}

Let us recall some nonlinear estimates in weighted Besov spaces from [12]. 
Lemma 2.5 Let $1 \leq p, q, q_{1}, q_{2} \leq \infty$ with $\frac{1}{q_{1}}+\frac{1}{q_{2}}=\frac{1}{q}$. Then there hold (a) if $s_{2} \leq \frac{3}{p}$, we have

$$
\left\|T_{g} f\right\|_{\widetilde{L}_{T}^{q}\left(\dot{B}_{p, 1}^{s_{1}+s_{2}-\frac{3}{p}}(\omega)\right)} \leq C\|f\|_{\widetilde{L}_{T}^{q_{1}}\left(\dot{B}_{p, 1}^{s_{1}}(\omega)\right)}\|g\|_{\widetilde{L}_{T}^{q_{2}}\left(\dot{B}_{p, 1}^{s_{2}}\right)} ;
$$

(b) if $s_{1} \leq \frac{3}{p}-1$, we have

$$
\left\|T_{f} g\right\|_{\widetilde{L}_{T}^{q}\left(\dot{B}_{p, 1}^{s_{1}+s_{2}-\frac{3}{p}}(\omega)\right)} \leq C\|f\|_{\widetilde{L}_{T}^{q_{1}}\left(\dot{B}_{p, 1}^{s_{1}}(\omega)\right)}\|g\|_{\widetilde{L}_{T}^{q_{2}}\left(\dot{B}_{p, 1}^{s_{2}}\right)} ;
$$

(c) if $s_{1}+s_{2}>3 \max \left(0, \frac{2}{p}-1\right)$, we have

$$
\|R(f, g)\|_{\widetilde{L}_{T}^{q}\left(\dot{B}_{p, 1}^{s_{1}+s_{2}-\frac{3}{p}}(\omega)\right)} \leq C\|f\|_{\widetilde{L}_{T}^{q_{1}}\left(\dot{B}_{p, 1}^{s_{1}}(\omega)\right)}\|g\|_{\widetilde{L}_{T}^{q_{2}}\left(\dot{B}_{p, 1}^{s_{2}}\right)} .
$$

The following lemma is a direct consequence of Lemma 2.5.

Lemma 2.6 Let $s_{1} \leq \frac{3}{p}-1, s_{2} \leq \frac{3}{p}, s_{1}+s_{2}>3 \max \left(0, \frac{2}{p}-1\right)$, and $1 \leq p, q, q_{1}, q_{2} \leq \infty$ with $\frac{1}{q_{1}}+\frac{1}{q_{2}}=\frac{1}{q}$. Then there holds

$$
\|f g\|_{\widetilde{L}_{T}^{q}\left(\dot{B}_{p, 1}^{s_{1}+s_{2}-\frac{3}{p}}(\omega)\right)} \leq C\|f\|_{\widetilde{L}_{T}^{q_{1}}\left(\dot{B}_{p, 1}^{s_{1}}(\omega)\right)}\|g\|_{\widetilde{L}_{T}^{q_{2}}\left(\dot{B}_{p, 1}^{s_{2}}\right)} .
$$

In general Besov spaces, we have

Lemma 2.7 Let $s_{1}, s_{2} \leq \frac{3}{p}, s_{1}+s_{2}>3 \max \left(0, \frac{2}{p}-1\right)$, and $1 \leq p, q, q_{1}, q_{2} \leq \infty$ with $\frac{1}{q_{1}}+\frac{1}{q_{2}}=$ $\frac{1}{q}$. Then there holds

$$
\|f g\|_{\widetilde{L}_{T}^{q}\left(\dot{B}_{p, 1}^{\left.s_{1}+s_{2}-\frac{3}{p}\right)}\right.} \leq C\|f\|_{\widetilde{L}_{T}^{q_{1}}\left(\dot{B}_{p, 1}^{s_{1}}\right)}\|g\|_{\widetilde{L}_{T}^{q_{2}}\left(\dot{B}_{p, 1}^{s_{2}}\right)}
$$

Lemma 2.8 Let $s>0$ and $1 \leq p, q, r \leq \infty$. Assume that $F \in W_{l o c}^{[s]+3, \infty}(\mathbf{R})$ with $F(0)=0$. Then there holds

$$
\|F(f)\|_{\widetilde{L}_{T}^{q}\left(\dot{B}_{p, r}^{s}(\omega)\right)} \leq C\left(1+\|f\|_{L_{T}^{\infty}\left(L^{\infty}\right)}\right)^{[s]+2}\|f\|_{\widetilde{L}_{T}^{q}\left(\dot{B}_{p, r}^{s}(\omega)\right)} .
$$

The same result also holds true in Besov space without weight.

The following is a weighted commutator estimate.

Lemma 2.9 Let $p \in[1, \infty)$ and $s \in\left(-3 \min \left(\frac{1}{p}, \frac{1}{p^{\prime}}\right), \frac{3}{p}\right]$. Then there holds

$$
\left\|2^{j s} \omega_{j}(T)\right\|\left[\Delta_{j}, f\right] \nabla g\left\|_{L_{T}^{1}\left(L^{p}\right)}\right\|_{\ell^{1}} \leq C\|f\|_{\widetilde{L}_{T}^{\infty}\left(\dot{B}_{p, 1}^{\frac{3}{p}}(\omega)\right)}\|g\|_{\widetilde{L}_{T}^{1}\left(\dot{B}_{p, 1}^{s+1}\right)} .
$$

Proof. Using Bony's decomposition (2.2), we write

$$
\begin{aligned}
{\left[f, \Delta_{j}\right] \cdot \partial_{k} g=} & {\left[T_{f}, \Delta_{j}\right] \partial_{k} g+T_{\partial_{k} \Delta_{j} g} f+R\left(f, \partial_{k} \Delta_{j} g\right) } \\
& -\Delta_{j}\left(T_{\partial_{k} g} f\right)-\Delta_{j} R\left(f, \partial_{k} g\right) .
\end{aligned}
$$


Using Lemma 2.5 (a) and (c) with $s_{1}=\frac{3}{p}$ and $s_{2}=s$, we get

$$
\begin{aligned}
& \left\|\omega_{j}(T) 2^{j s}\right\| \Delta_{j}\left(T_{\partial_{k} g} f\right)\left\|_{L_{T}^{1}\left(L^{p}\right)}\right\|_{\ell^{1}} \leq C\|f\|_{\widetilde{L}_{T}^{\infty}\left(\dot{B}_{p, 1}^{\frac{3}{p}}(\omega)\right)}\|g\|_{\widetilde{L}_{T}^{1}\left(\dot{B}_{p, 1}^{s+1}\right)}, \\
& \left\|\omega_{j}(T) 2^{j s}\right\| \Delta_{j} R\left(f^{k}, \partial_{k} g\right)\left\|_{L_{T}^{1}\left(L^{p}\right)}\right\|_{\ell^{1}} \leq C\|f\|_{\widetilde{L}_{T}^{\infty}\left(\dot{B}_{p, 1}^{\frac{3}{p}}(\omega)\right)}\|g\|_{\widetilde{L}_{T}^{1}\left(\dot{B}_{p, 1}^{s+1}\right)} .
\end{aligned}
$$

Noticing that

$$
T_{\partial_{k} \Delta_{j} g}^{\prime} f \triangleq T_{\partial_{k} \Delta_{j} g} f+R\left(f, \partial_{k} \Delta_{j} g\right)=\sum_{j^{\prime} \geq j-2} S_{j^{\prime}+2} \Delta_{j} \partial_{k} g \Delta_{j^{\prime}} f
$$

then we get by Lemma 2.1 and (2.4) that

$$
\begin{aligned}
& \left\|\omega_{j}(T) 2^{j s}\right\| T_{\partial_{k} \Delta_{j} g}^{\prime} f\left\|_{L_{T}^{1}\left(L^{p}\right)}\right\|_{\ell^{1}} \\
& \leq C\left\|\omega_{j}(T) 2^{j s}\right\| \Delta_{j} \nabla g\left\|_{L_{T}^{1}\left(L^{\infty}\right)} \sum_{j^{\prime} \geq j-2}\right\| \Delta_{j^{\prime}} f\left\|_{L_{T}^{\infty}\left(L^{p}\right)}\right\|_{\ell^{1}} \\
& \leq C\left\|2^{j\left(s+1+\frac{3}{p}\right)}\right\| \Delta_{j} g\left\|_{L_{T}^{1}\left(L^{p}\right)} \sum_{j^{\prime} \geq j-2} \omega_{j^{\prime}}(T)\right\| \Delta_{j^{\prime}} f\left\|_{L_{T}^{\infty}\left(L^{p}\right)}\right\|_{\ell^{1}} \\
& \leq C\|f\|_{\widetilde{L}_{T}^{\infty}\left(\dot{B}_{p, 1}^{\frac{3}{p}}(\omega)\right)}\|g\|_{\widetilde{L}_{T}^{1}\left(\dot{B}_{p, 1}^{s+1}\right)} \cdot
\end{aligned}
$$

Set $h(x)=\left(\mathcal{F}^{-1} \varphi\right)(x)$. Then we have

$$
\begin{gathered}
{\left[T_{f}, \Delta_{j}\right] \partial_{k} g=\sum_{\left|j^{\prime}-j\right| \leq 4} 2^{4 j} \int_{\mathbf{R}^{3}} \int_{0}^{1} y \cdot \nabla S_{j^{\prime}-1} f(x-\tau y) d \tau \partial_{k} h\left(2^{j} y\right) \Delta_{j^{\prime}} g(x-y) d y} \\
+2^{3 j} \int_{\mathbf{R}^{3}} h\left(2^{j}(x-y)\right) \partial_{k} S_{j^{\prime}-1} f(y) \Delta_{j^{\prime}} g(y) d y
\end{gathered}
$$

from which and Young's inequality, we infer that

$$
\left\|\omega_{j}(T) 2^{j s}\right\|\left[T_{f}, \Delta_{j}\right] \partial_{k} g\left\|_{L_{T}^{1}\left(L^{p}\right)}\right\|_{\ell^{1}} \leq C\|f\|_{\widetilde{L}_{T}^{\infty}\left(\dot{B}_{p, 1}^{\frac{3}{p}}(\omega)\right)}\|g\|_{\widetilde{L}_{T}^{1}\left(\dot{B}_{p, 1}^{s+1}\right)} .
$$

The proof is finished by summing up all the above estimates.

\section{The linear transport equation and momentum equation}

We consider the linear transport equation

$$
\left\{\begin{array}{l}
\partial_{t} f+v \cdot \nabla f=g, \\
f(0, x)=f_{0}
\end{array}\right.
$$

Set $V(t) \stackrel{\text { def }}{=} \int_{0}^{t}\|\nabla v(\tau)\|_{\dot{B}_{p, 1}^{\frac{3}{p}}} d \tau$. The following result is from [18]. 
Proposition 3.1 Let $p \in[1,+\infty]$ and $s \in\left(-3 \min \left(\frac{1}{p}, \frac{1}{p^{\prime}}\right), 1+\frac{3}{p}\right)$. Let $v$ be a vector field such that $\nabla v \in L_{T}^{1}\left(\dot{B}_{p, 1}^{\frac{3}{p}}\right)$. Assume that $f_{0} \in \dot{B}_{p, 1}^{s}, g \in L_{T}^{1}\left(\dot{B}_{p, 1}^{s}\right)$ and $f$ is the solution of (3.1). Then there holds for $t \in[0, T]$,

$$
\|f\|_{\widetilde{L}_{t}^{\infty}\left(\dot{B}_{p, 1}^{s}\right)} \leq e^{C V(t)}\left(\left\|f_{0}\right\|_{\dot{B}_{p, 1}^{s}}+\int_{0}^{t} e^{-C V(\tau)}\|g(\tau)\|_{\dot{B}_{p, 1}^{s}} d \tau\right)
$$

The following weighted version is from [12].

Proposition 3.2 Let $p \in[1,+\infty]$ and $s \in\left(-3 \min \left(\frac{1}{p}, \frac{1}{p^{\prime}}\right), \frac{3}{p}\right]$. Let $v$ be a vector field such that $\nabla v \in L_{T}^{1}\left(\dot{B}_{p, 1}^{\frac{3}{p}}\right)$. Assume that $f_{0} \in \dot{B}_{p, 1}^{s}, g \in L_{T}^{1}\left(\dot{B}_{p, 1}^{s}\right)$ and $f$ is the solution of (3.1). Then there holds for $t \in[0, T]$,

$$
\|f\|_{\widetilde{L}_{t}^{\infty}\left(\dot{B}_{p, 1}^{s}(\omega)\right)} \leq e^{C V(t)}\left(\left\|f_{0}\right\|_{\dot{B}_{p, 1}^{s}(\omega)}+\int_{0}^{t} e^{-C V(\tau)}\|g(\tau)\|_{\dot{B}_{p, 1}^{s}(\omega)} d \tau\right) .
$$

We next study the linear momentum equations with variable coefficients

$$
\left\{\begin{array}{l}
\partial_{t} u-\operatorname{div}(\bar{\mu} \nabla u)-\nabla(\bar{\lambda} \operatorname{div} u)=G \\
\left.u\right|_{t=0}=u_{0}
\end{array}\right.
$$

Assume that the viscosity coefficients $\bar{\lambda}(\rho)$ and $\bar{\mu}(\rho)$ depend smoothly on the function $\rho$ and there exists a positive constant $c_{3}$ such that

$$
\bar{\mu} \geq c_{3}, \quad \bar{\lambda}+\bar{\mu} \geq c_{3} .
$$

And the weighted function $\omega_{k}(t)$ is taken as follows

$$
\omega_{k}(t)=\sum_{\ell \geq k} 2^{k-\ell} e_{\ell}(t)
$$

with $e_{\ell}(t)=\left(1-e^{-c 2^{2 \ell} t}\right)^{\frac{1}{2}}$ for some positive constant $c>0$. It is easy to verify that the function $e_{\ell}(t)$ satisfies (2.3).

Proposition 3.3 Let $p \in[2, \infty), s \in\left(1-\frac{3}{p}, \frac{3}{p}\right], q \in[1, \infty]$. Assume that $u_{0} \in \dot{B}_{p, 1}^{s-1}, G \in$ $L_{T}^{1}\left(\dot{B}_{p, 1}^{s-1}\right)$, and $\rho-\bar{\rho} \in \widetilde{L}_{T}^{\infty}\left(\dot{B}_{p, 1}^{\frac{3}{p}}\right)$. Let $u$ be a solution of (3.2). Then we have

$$
\begin{aligned}
& \|u\|_{\widetilde{L}_{T}^{1}\left(\dot{B}_{p, 1}^{s+1}\right)}+\|u\|_{\widetilde{L}_{T}^{2}\left(\dot{B}_{p, 1}^{s}\right)} \\
& \leq C\left(\left\|u_{0}\right\|_{\left.\dot{B}_{p, 1}^{s-1}(\omega)\right)}+\|G\|_{\widetilde{L}_{T}^{1}\left(\dot{B}_{p, 1}^{s-1}(\omega)\right)}+A(T)\|\rho-\bar{\rho}\|_{\widetilde{L}_{T}^{\infty}\left(\dot{B}_{p, 1}^{\frac{3}{p}}(\omega)\right)}\|u\|_{\widetilde{L}_{T}^{1}\left(\dot{B}_{p, 1}^{s+1}\right)}\right),
\end{aligned}
$$

and the version without weight

$$
\|u\|_{\widetilde{L}_{T}^{q}\left(\dot{B}_{p, 1}^{\left.s-1+\frac{2}{q}\right)}\right.} \leq C\left(\left\|u_{0}\right\|_{\dot{B}_{p, 1}^{s-1}}+\|G\|_{\widetilde{L}_{T}^{1}\left(\dot{B}_{p, 1}^{s-1}\right)}+A(T)\left\|\rho-\bar{\rho}_{\widetilde{L}_{T}^{\infty}\left(\dot{B}_{p, 1}^{\frac{3}{p}}\right)}\right\| u \|_{\widetilde{L}_{T}^{(}\left(\dot{B}_{p, 1}^{s+1}\right)}\right) .
$$

Here $A(T) \stackrel{\text { def }}{=}\left(1+\|\rho\|_{L_{T}^{\infty}\left(L^{\infty}\right)}\right)^{\left[\frac{3}{p}\right]+2}$. 
Proof. We only prove the first inequality, the proof of the second inequality is similar. Set $d=\operatorname{div} u$ and $w=\operatorname{curl} u$. By (3.2),$(d, w)$ satisfies

$$
\left\{\begin{array}{l}
\partial_{t} d-\operatorname{div}(\bar{\nu} \nabla d)=\operatorname{div} G+F_{1}, \\
\partial_{t} w-\operatorname{div}(\bar{\mu} \nabla w)=\operatorname{curl} G+F_{2}, \\
\left.(d, w)\right|_{t=0}=\left(\operatorname{div} u_{0}, \operatorname{curl} u_{0}\right) \triangleq\left(d_{0}, w_{0}\right),
\end{array}\right.
$$

where $\bar{\nu}=\bar{\lambda}+\bar{\mu}$ and

$$
\begin{aligned}
& F_{1}=\operatorname{div}(\nabla \bar{\mu} \cdot \nabla u)+\operatorname{div}(\nabla(\bar{\lambda}+\bar{\mu}) d), \\
& F_{2}^{i, j}=\operatorname{div}\left(\partial_{j} \bar{\mu} \nabla u^{i}-\partial_{i} \bar{\mu} \nabla u^{j}\right), \quad i, j=1,2,3 .
\end{aligned}
$$

Apply the operator $\Delta_{j}$ to (3.3) to obtain

$$
\left\{\begin{array}{l}
\partial_{t} \Delta_{j} d-\operatorname{div}\left(\bar{\nu} \nabla \Delta_{j} d\right)=\operatorname{div} \Delta_{j} G+\Delta_{j} F_{1}+\operatorname{div}\left[\Delta_{j}, \bar{\nu}\right] \nabla d \\
\partial_{t} \Delta_{j} w-\operatorname{div}\left(\bar{\mu} \nabla \Delta_{j} w\right)=\operatorname{curl} \Delta_{j} G+\Delta_{j} F_{2}+\operatorname{div}\left[\Delta_{j}, \bar{\mu}\right] \nabla w
\end{array}\right.
$$

Multiplying the first equation by $\left|\Delta_{j} d\right|^{p-2} \Delta_{j} d$, we get by integrating over $\mathbf{R}^{3}$ that

$$
\begin{aligned}
\frac{1}{p} & \frac{d}{d t}\left\|\Delta_{j} d\right\|_{p}^{p}-\int_{\mathbf{R}^{3}} \operatorname{div}\left(\bar{\nu} \nabla \Delta_{j} d\right)\left|\Delta_{j} d\right|^{p-2} \Delta_{j} d \mathrm{~d} x \\
\quad & =\int_{\mathbf{R}^{3}}\left(\operatorname{div} \Delta_{j} G+\Delta_{j} F_{1}+\operatorname{div}\left[\Delta_{j}, \bar{\nu}\right] \nabla d\right)\left|\Delta_{j} d\right|^{p-2} \Delta_{j} d d x .
\end{aligned}
$$

There exists $c_{p}>0$ depending on $c_{3}, p$ such that(see [16])

$$
\frac{1}{p} \frac{d}{d t}\left\|\Delta_{j} d\right\|_{p}^{p}+c_{p} 2^{2 j}\left\|\Delta_{j} d\right\|_{p}^{p} \leq C\left\|\Delta_{j} d\right\|_{p}^{p-1}\left(2^{j}\left\|\Delta_{j} G\right\|_{p}+\left\|\Delta_{j} F_{1}\right\|_{p}+2^{j}\left\|\left[\Delta_{j}, \bar{\nu}\right] \nabla d\right\|_{p}\right) .
$$

This gives that

$$
\frac{d}{d t}\left\|\Delta_{j} d\right\|_{p}+c_{p} 2^{2 j}\left\|\Delta_{j} d\right\|_{p} \leq C\left(2^{j}\left\|\Delta_{j} G\right\|_{p}+\left\|\Delta_{j} F_{1}\right\|_{p}+2^{j}\left\|\left[\Delta_{j}, \bar{\nu}\right] \nabla d\right\|_{p}\right),
$$

which implies that

$$
\left\|\Delta_{j} d(t)\right\|_{p} \leq e^{-c_{p} 2^{2 j} t}\left\|\Delta_{j} d_{0}\right\|_{p}+C \int_{0}^{t} e^{-c_{p} 2^{2 j}(t-\tau)}\left(2^{j}\left\|\Delta_{j} G\right\|_{p}+\left\|\Delta_{j} F_{1}\right\|_{p}+2^{j}\left\|\left[\Delta_{j}, \bar{\nu}\right] \nabla d\right\|_{p}\right) d \tau .
$$

Similarly, we can show that

$$
\left\|\Delta_{j} w(t)\right\|_{p} \leq e^{-c_{p} 2^{2 j} t}\left\|\Delta_{j} d_{0}\right\|_{p}+C \int_{0}^{t} e^{-c_{p} 2^{2 j}(t-\tau)}\left(2^{j}\left\|\Delta_{j} G\right\|_{p}+\left\|\Delta_{j} F_{2}\right\|_{p}+2^{j}\left\|\left[\Delta_{j}, \bar{\mu}\right] \nabla w\right\|_{p}\right) d \tau .
$$

Hence, we infer that for any $q \in[1, \infty]$ and $t \in[0, T]$,

$$
\begin{aligned}
& \left\|\Delta_{j} d(t)\right\|_{L_{t}^{q}\left(L^{p}\right)}+\left\|\Delta_{j} w(t)\right\|_{L_{t}^{q}\left(L^{p}\right)} \\
& \leq C 2^{-2 j / q} c_{j}(T)^{\frac{1}{q}}\left(\left\|\Delta_{j} d_{0}\right\|_{p}+\left\|\Delta_{j} w_{0}\right\|_{p}\right) \\
& \quad+C 2^{-2 j / q} c_{j}(T)^{\frac{1}{q}}\left(2^{j}\left\|\Delta_{j} G\right\|_{L_{t}^{1}\left(L^{p}\right)}+\left\|\Delta_{j} F_{1}\right\|_{L_{t}^{1}\left(L^{p}\right)}+2^{j}\left\|\left[\Delta_{j}, \bar{\nu}\right] \nabla d\right\|_{L_{t}^{1}\left(L^{p}\right)}\right) \\
& \quad+C 2^{-2 j / q} c_{j}(T)^{\frac{1}{q}}\left(2^{j}\left\|\Delta_{j} G\right\|_{L_{t}^{1}\left(L^{p}\right)}+\left\|\Delta_{j} F_{2}\right\|_{L_{t}^{1}\left(L^{p}\right)}+2^{j}\left\|\left[\Delta_{j}, \bar{\mu}\right] \nabla w\right\|_{L_{t}^{1}\left(L^{p}\right)}\right),
\end{aligned}
$$


with $c_{j}(T)=1-e^{-c_{p} 2^{2 j} T}$. Notice that

$$
2^{j}\left\|\Delta_{j} u\right\|_{p} \sim\left\|\Delta_{j} d\right\|_{p}+\left\|\Delta_{j} w\right\|_{p}, \quad e_{j}(T) \leq \omega_{j}(T),
$$

which along with (3.4) implies that

$$
\begin{aligned}
& \|u\|_{\widetilde{L}_{T}^{1}\left(\dot{B}_{p, 1}^{s+1}\right)}+\|u\|_{\widetilde{L}_{T}^{2}\left(\dot{B}_{p, 1}^{s}\right)} \\
& \leq C\left(\left\|u_{0}\right\|_{\dot{B}_{p, 1}^{s-1}(\omega)}+\|G\|_{\widetilde{L}_{T}^{1}\left(\dot{B}_{p, 1}^{s-1}(\omega)\right)}+\left\|\left(F_{1}, F_{2}\right)\right\|_{\widetilde{L}_{T}^{1}\left(\dot{B}_{p, 1}^{s-2}(\omega)\right)}\right) \\
& \quad+C\left\|2^{j(s-1)} w_{j}(T)\left(\left\|\left[\Delta_{j}, \bar{\nu}\right] \nabla d\right\|_{L_{T}^{1}\left(L^{p}\right)}+\left\|\left[\Delta_{j}, \bar{\mu}\right] \nabla w\right\|_{L_{T}^{1}\left(L^{p}\right)}\right)\right\|_{\ell^{1}} .
\end{aligned}
$$

From Lemma 2.6 and Lemma 2.8, it follows that

$$
\left\|F_{1}\right\|_{\widetilde{L}_{T}^{1}\left(\dot{B}_{p, 1}^{s-2}(\omega)\right)}+\left\|F_{2}\right\|_{\widetilde{L}_{T}^{1}\left(\dot{B}_{p, 1}^{s-2}(\omega)\right)} \leq A(T)\|\rho-\bar{\rho}\|_{\widetilde{L}_{T}^{\infty}\left(\dot{B}_{p, 1}^{\frac{3}{p}}(\omega)\right)}\|u\|_{\widetilde{L}_{T}^{1}\left(\dot{B}_{p, 1}^{s+1}\right)},
$$

and using Lemma 2.9, we get

$$
\begin{aligned}
& \left\|2^{j(s-1)} \omega_{j}(T)\left(\left\|\left[\Delta_{j}, \bar{\nu}\right] \nabla d\right\|_{L_{T}^{1}\left(L^{p}\right)}+\left\|\left[\Delta_{j}, \bar{\mu}\right] \nabla w\right\|_{L_{T}^{1}\left(L^{p}\right)}\right)\right\|_{\ell^{1}} \\
& \leq A(T)\|\rho-\bar{\rho}\|_{\widetilde{L}_{T}^{\infty}\left(\dot{B}_{p, 1}^{\frac{3}{p}}(\omega)\right)}\|u\|_{\widetilde{L}_{T}^{1}\left(\dot{B}_{p, 1}^{s+1}\right)} .
\end{aligned}
$$

Summing up (3.5)-(3.7) yields the first inequality of Proposition 3.3.

\section{Local well-posedness with physical non vacuum}

In this section, we prove the local well-posedness of compressible Navier-Stokes equations in the critical Besov space with physical non vacuum assumption. That is, we just impose $\rho_{0} \geq c_{0} 0$ for the initial density. In the case when $\rho-\bar{\rho}_{0}$ is small in $\dot{B}_{p, 1}^{\frac{3}{p}}$ or has more regularity, the corresponding local-well posedness has been obtained by Danchin [16]. Recently, ChenMiao-Zhang [12] and Danchin [19] developed two different methods to deal with physical non vacuum case. Here we will revisit the proof of [12]. The main goal is to record the precise dependence of energy bounds and the existence time $T$ on the initial data and other important constants, which is very important for our argument.

Theorem 4.1 Let $\bar{\rho}>0$ and $c_{0}>0$. Assume that the initial data $\left(\rho_{0}, u_{0}\right)$ satisfies

$$
\rho_{0}-\bar{\rho} \in \dot{B}_{p, 1}^{\frac{3}{p}}, \quad c_{0} \leq \rho_{0} \leq c_{0}^{-1}, \quad u_{0} \in \dot{B}_{p, 1}^{\frac{3}{p}-1} .
$$

Then there exists a positive time $T$ such that

(a) Existence: If $p \in[2,6)$, the system (1.1) has a solution $(\rho-\bar{\rho}, u) \in E_{T}^{p}$ with

$$
E_{T}^{p} \stackrel{\text { def }}{=} C\left([0, T] ; \dot{B}_{p, 1}^{\frac{3}{p}}\right) \times\left(C\left([0, T] ; \dot{B}_{p, 1}^{\frac{3}{p}-1}\right) \cap L^{1}\left(0, T ; \dot{B}_{p, 1}^{\frac{3}{p}+1}\right)\right)^{3}, \quad \rho \geq \frac{1}{2} c_{0}
$$

(b) Uniqueness: If $p \in(1,3]$, then the uniqueness holds in $E_{T}^{p}$. 
Proof. We only present the uniform energy estimates for smooth solution of (1.1), since the existence can be deduced by constructing approximate solution sequence and compact argument. The uniqueness has been proved in [12]. Set

$$
a(t, x)=\frac{\rho(t, x)-\bar{\rho}}{\bar{\rho}}, \quad \bar{\mu}(\rho)=\frac{\mu}{\rho}, \quad \bar{\lambda}(\rho)=\frac{\lambda}{\rho} .
$$

Then the system (1.1) can be rewritten as

$$
\left\{\begin{array}{l}
\partial_{t} a+u \cdot \nabla a=F \\
\partial_{t} u-\operatorname{div}(\bar{\mu} \nabla u)-\nabla(\bar{\lambda} \operatorname{div} u)=G \\
\left.(a, u)\right|_{t=0}=\left(a_{0}, u_{0}\right)
\end{array}\right.
$$

with $a_{0}=\frac{\rho_{0}(x)-\bar{\rho}}{\bar{\rho}}$ and

$$
\begin{aligned}
& F(=-(1+a) \operatorname{div} u \\
& G=-u \cdot \nabla u-\frac{\bar{\rho} P^{\prime}(\rho)}{\rho} \nabla a+\frac{\mu}{\rho^{2}} \nabla \rho \cdot \nabla u+\frac{\lambda}{\rho^{2}} \nabla \rho \operatorname{div} u .
\end{aligned}
$$

Let us assume that the solution $(\rho, u)$ satisfies

$$
\begin{aligned}
& \frac{c_{0}}{2} \leq \rho(t, x) \leq 2 c_{0}^{-1} \quad \text { on } \quad[0, T] ; \\
& \|a\|_{\widetilde{L}_{T}^{\infty}\left(\dot{B}_{p, 1}^{\frac{3}{p}}\right)}+\|u\|_{\widetilde{L}_{T}^{\infty}\left(\dot{B}_{p, 1}^{\frac{3}{p}-1}\right)} \leq C_{0}\left(\left\|a_{0}\right\|_{\dot{B}_{p, 1}^{\frac{3}{p}}}+\left\|u_{0}\right\|_{\dot{B}_{p, 1}^{\frac{3}{p}-1}}\right) \stackrel{\text { def }}{=} C_{0} E_{0} ; \\
& \|a\|_{\widetilde{L}_{T}^{\infty}\left(\dot{B}_{p, 1}^{\frac{3}{p}}(\omega)\right)} \leq \eta_{1} ; \\
& \|u\|_{\widetilde{L}_{T}^{1}\left(\dot{B}_{p, 1}^{\frac{3}{p}}\right)}+\|u\|_{\widetilde{L}_{T}^{2}\left(\dot{B}_{p, 1}^{\frac{3}{p}}\right)} \leq \eta_{2} .
\end{aligned}
$$

These assumptions are satisfied for the solution in $E_{T}^{p}$ if $T$ is small enough. We will show that there exists a $T>0$ depending on the initial data and $\lambda, \mu, \bar{\rho}, c_{0}, p$ such that these assumptions are satisfied.

In what follows, we denote $C$ by a constant depending only on $\lambda, \mu, \bar{\rho}, c_{0}, p$, which may be different from line to line.

Step 1. Estimates in the Besov space

Applying Proposition 3.1 to the first equation of (4.2), we get

$$
\|a\|_{\widetilde{L}_{T}^{\infty}\left(\dot{B}_{p, 1}^{\frac{3}{p}}\right)} \leq e^{C V(T)}\left(\left\|a_{0}\right\|_{\dot{B}_{p, 1}^{\frac{3}{p}}}+\|F\|_{\widetilde{L}_{T}^{1}\left(\dot{B}_{p, 1}^{\frac{3}{p}}\right)}\right),
$$

and applying Proposition 3.3 to the second equation of (4.2), we have

$$
\|u\|_{\widetilde{L}_{T}^{\infty}\left(\dot{B}_{p, 1}^{\frac{3}{p}-1}\right)} \leq C\left\|u_{0}\right\|_{\dot{B}_{p, 1}^{\frac{3}{p}-1}}+C\|G\|_{\widetilde{L}_{T}^{1}\left(\dot{B}_{p, 1}^{\frac{3}{p}-1}\right)}+C\|a\|_{\widetilde{L}_{T}^{\infty}\left(\dot{B}_{p, 1}^{\frac{3}{p}}\right)}\|u\|_{\widetilde{L}_{T}^{1}\left(\dot{B}_{p, 1}^{\frac{3}{p}+1}\right)},
$$

where $V(t)=\int_{0}^{t}\|u(\tau)\|_{\dot{B}_{p, 1}^{\frac{3}{p}+1}} d \tau$. By Lemma 2.7, we have

$$
\|F\|_{\widetilde{L}_{T}^{1}\left(\dot{B}_{p, 1}^{\frac{3}{p}}\right)} \leq\|u\|_{\widetilde{L}_{T}^{1}\left(\dot{B}_{p, 1}^{\frac{3}{p}+1}\right)}+C\|a\|_{\widetilde{L}_{T}^{\infty} \dot{B}_{p, 1}^{\frac{3}{p}}}\|u\|_{\widetilde{L}_{T}^{1}\left(\dot{B}_{p, 1}^{\frac{3}{p}+1}\right)},
$$


and by Lemma 2.7 and Lemma 2.8 ,

$$
\|G\|_{\widetilde{L}_{T}^{1}\left(\dot{B}_{p, 1}^{\frac{3}{p}-1}\right)} \leq C\|u\|_{\widetilde{L}_{T}^{\infty}\left(\dot{B}_{p, 1}^{\frac{3}{p}-1}\right)}\|u\|_{\widetilde{L}_{T}^{1}\left(\dot{B}_{p, 1}^{\frac{3}{p}+1}\right)}+C\|a\|_{\widetilde{L}_{T}^{\infty}\left(\dot{B}_{p, 1}^{\frac{3}{p}}\right)}\left(T+\|u\|_{\widetilde{L}_{T}^{1}\left(\dot{B}_{p, 1}^{\frac{3}{p}+1}\right)}\right) .
$$

Here we used $p<6$. Plugging them into (4.7)-(4.8), we obtain

$$
\|a\|_{\widetilde{L}_{T}^{\infty}\left(\dot{B}_{p, 1}^{\frac{3}{p}}\right)}+\|u\|_{\widetilde{L}_{T}^{\infty}\left(\dot{B}_{p, 1}^{\frac{3}{p}-1}\right)} \leq C e^{C \eta_{2}}\left(E_{0}+C_{0} E_{0}\left(\eta_{2}+T\right)+\eta_{2}\right) .
$$

Step 2. Estimates in the weighted Besov space

Applying Proposition 3.2 to the first equation of (4.2), we get

$$
\|a\|_{\widetilde{L}_{T}^{\infty}\left(\dot{B}_{p, 1}^{\frac{3}{p}}(\omega)\right)} \leq e^{C V(T)}\left(\left\|a_{0}\right\|_{\dot{B}_{p, 1}^{\frac{3}{p}}(\omega)}+\|F\|_{\widetilde{L}_{T}^{1}\left(\dot{B}_{p, 1}^{\frac{3}{p}}(\omega)\right)}\right),
$$

and by Proposition 3.3, we have

$$
\begin{aligned}
& \|u\|_{\widetilde{L}_{T}^{1}\left(\dot{B}_{p, 1}^{\frac{3}{p}+1}\right)}+\|u\|_{\widetilde{L}_{T}^{2}\left(\dot{B}_{p, 1}^{\frac{3}{p}}\right)} \\
& \leq C\left(\left\|u_{0}\right\|_{\left.\dot{B}_{p, 1}^{\frac{3}{p}-1}(\omega)\right)}+\|G\|_{\widetilde{L}_{T}^{1}\left(\dot{B}_{p, 1}^{\frac{3}{p}-1}(\omega)\right)}+\|a\|_{\widetilde{L}_{T}^{\infty}\left(\dot{B}_{p, 1}^{\frac{3}{p}}(\omega)\right)}\|u\|_{\widetilde{L}_{T}^{1}\left(\dot{B}_{p, 1}^{\frac{3}{p}+1}\right)}\right) .
\end{aligned}
$$

By Lemma 2.7, we have

$$
\|F\|_{\widetilde{L}_{T}^{1}\left(\dot{B}_{p, 1}^{\frac{3}{p}}(\omega)\right)} \leq 2\|F\|_{\widetilde{L}_{T}^{1}\left(\dot{B}_{p, 1}^{\frac{3}{p}}\right)} \leq C\left(1+\|a\|_{\widetilde{L}_{T}^{\infty}\left(\dot{B}_{p, 1}^{\frac{3}{p}}\right)}\|u\|_{\widetilde{L}_{T}^{1}\left(\dot{B}_{p, 1}^{\frac{3}{p}+1}\right)},\right.
$$

and by Lemma 2.6 and Lemma 2.8 ,

$$
\|G\|_{\widetilde{L}_{T}^{1}\left(\dot{B}_{p, 1}^{\frac{3}{p}-1}(\omega)\right)} \leq C\|u\|_{\widetilde{L}_{T}^{2}\left(\dot{B}_{p, 1}^{\frac{3}{p}}\right)}^{2}+C\|a\|_{\widetilde{L}_{T}^{\infty}\left(\dot{B}_{p, 1}^{\frac{3}{p}}(\omega)\right)}\left(T+\|u\|_{\widetilde{L}_{T}^{1}\left(\dot{B}_{p, 1}^{\frac{3}{p}+1}\right)}\right) .
$$

Plugging them into (4.10)-(4.11) yields that

$$
\begin{aligned}
& \|a\|_{\widetilde{L}_{T}^{\infty}\left(\dot{B}_{p, 1}^{\frac{3}{p}}(\omega)\right)} \leq C e^{C_{1} \eta_{2}}\left(\left\|a_{0}\right\|_{\dot{B}_{p, 1}^{\frac{3}{p}}(\omega)}+\left(1+C_{0} E_{0}\right) \eta_{2}\right), \\
& \|u\|_{\widetilde{L}_{T}^{1}\left(\dot{B}_{p, 1}^{\frac{3}{p}+1}\right)}+\|u\|_{\widetilde{L}_{T}^{2}\left(\dot{B}_{p, 1}^{\frac{3}{p}}\right)} \leq C\left(\left\|u_{0}\right\|_{\left.\dot{B}_{p, 1}^{\frac{3}{p}-1}(\omega)\right)}+\eta_{2}^{2}+\eta_{1}\left(T+\eta_{2}\right)\right) .
\end{aligned}
$$

Step 3. Estimate of the density

Let $X(t, x)$ be a solution of

$$
\frac{d}{d t} X(t, x)=u(t, X(t, x)), \quad X(0, x)=x,
$$

and we denote by $X^{-1}(t, x)$ the inverse of $X(t, x)$. Then $a(t, x)$ can be solved as

$$
a(t, x)=a_{0}\left(X^{-1}(t, x)\right)+\int_{0}^{t} F\left(\tau, X\left(\tau, X^{-1}(t, x)\right)\right) d \tau,
$$

this gives

$$
\rho(t, x)=\rho_{0}\left(X^{-1}(t, x)\right)+\bar{\rho}_{0} \int_{0}^{t} F\left(\tau, X\left(\tau, X^{-1}(t, x)\right)\right) d \tau .
$$


Since we have

$$
\|F\|_{L_{T}^{1}\left(L^{\infty}\right)} \leq\|\nabla u\|_{L_{T}^{1}\left(L^{\infty}\right)}\left(1+\|a\|_{L_{T}^{\infty}\left(L^{\infty}\right)}\right) \leq C \eta_{2},
$$

from which and (4.14), it follows that

$$
\rho(t, x) \geq c_{0}-C \eta_{2}, \quad \rho(t, x) \leq c_{0}^{-1}+C \eta_{2} .
$$

Step 4. Continuity argument

Let $C_{0}=10 C$. We first take $\eta_{2}$ and $\eta_{1}$ small enough such that

$$
\begin{aligned}
& C \eta_{1} \leq \frac{1}{8}, \quad e^{C \eta_{2}} \leq 2, \quad C_{0} \eta_{2} \leq 1, \quad \eta_{2} \leq E_{0}, \\
& C \eta_{2} \leq \frac{c_{0}}{4}, \quad 2 C\left(1+C_{0} E_{0}\right) \eta_{2} \leq \frac{\eta_{1}}{4} .
\end{aligned}
$$

Then we take $T$ small enough such that

$$
C_{0} T \leq 1, \quad T \leq \eta_{2}, \quad\left\|a_{0}\right\|_{\dot{B}_{p, 1}^{\frac{3}{p}}(\omega)} \leq \frac{\eta_{1}}{4 C}, \quad\left\|u_{0}\right\|_{\dot{B}_{p, 1}^{\frac{3}{p}-1}(\omega)} \leq \frac{\eta_{2}}{4 C} .
$$

With this choice of $\eta_{1}, \eta_{2}$ and $T$, it follows from (4.9), (4.12), (4.13) and (4.15) that

$$
\begin{aligned}
& \|a\|_{\widetilde{L}_{T}^{\infty}\left(\dot{B}_{p, 1}^{\frac{3}{p}}\right)}+\|u\|_{\widetilde{L}_{T}^{\infty}\left(\dot{B}_{p, 1}^{\frac{3}{p}-1}\right)} \leq \frac{4}{5} C_{0} E_{0}, \\
& \|a\|_{\widetilde{L}_{T}^{\infty}\left(\dot{B}_{p, 1}^{\frac{3}{p}}(\omega)\right)} \leq \frac{3}{4} \eta_{1}, \\
& \|u\|_{\widetilde{L}_{T}^{1}\left(\dot{B}_{p, 1}^{\frac{3}{p}+1}\right)}+\|u\|_{\widetilde{L}_{T}^{2}\left(\dot{B}_{p, 1}^{\frac{3}{p}}\right)} \leq \frac{3}{4} \eta_{2}, \\
& \frac{3}{4} c_{0} \leq \rho(t, x) \leq \frac{5}{4} c_{0}^{-1}
\end{aligned}
$$

which implies that the assumptions (4.3)-(4.6) are satisfied for $T$ defined by (4.17).

Remark 4.2 In general, the choice of time $T$ depends on $C_{0}=C_{0}\left(\lambda, \mu, \bar{\rho}, c_{0}, p\right)$ and the behaviour of the initial data $\left(\rho_{0}, u_{0}\right)$. If $\left\|u_{0}\right\|_{\dot{B}_{p, 1}^{\frac{3}{p}-1}} \leq c$ and $a_{0} \in \dot{B}_{p, 1}^{\frac{3}{p}} \cap H^{s}$ for $s>\frac{3}{2}$ and $c=c\left(\lambda, \mu, \bar{\rho}, c_{0}, p\right)$ small enough, then $T$ can be taken as

$$
T=\frac{1}{C\left(1+\left\|a_{0}\right\|_{\dot{B}_{p, 1}^{\frac{3}{p}} \cap H^{s}}\right)^{\left(s+\frac{1}{2}\right) /\left(s-\frac{3}{2}\right)}}, \quad C=C\left(\lambda, \mu, \bar{\rho}, c_{0}, p, s\right) .
$$

Indeed, due to (4.16), we can take $\eta_{1}, \eta_{2}$ as follows

$$
\eta_{1}=\frac{1}{\widetilde{C}}, \quad \eta_{2}=\frac{1}{\widetilde{C}\left(1+\left\|a_{0}\right\|_{\dot{B}_{p, 1}^{\frac{3}{p}}}\right)} .
$$

for some constant $\widetilde{C}$ depending only on $\lambda, \mu, \bar{\rho}, c_{0}, p$. And by Lemma 2.1 , for any $N \in \mathbf{N}$,

$$
\sum_{j>N} 2^{j \frac{3}{p}}\left\|\Delta_{j} a_{0}\right\|_{p} \leq C \sum_{j>N} 2^{j \frac{3}{2}}\left\|\Delta_{j} a_{0}\right\|_{2} \leq C 2^{-\left(s-\frac{3}{2}\right) N}\left\|a_{0}\right\|_{H^{s}}
$$


And by 2.4, we have

$$
\sum_{j \leq N} 2^{j \frac{3}{p}} \omega_{j}(T)\left\|\Delta_{j} a_{0}\right\|_{p} \leq \omega_{N}(T)\left\|a_{0}\right\|_{\dot{B}_{p, 1}^{\frac{3}{p}}}
$$

Then (4.17) is ensured by taking $T$ as (4.18).

\section{Propagation of Sobolev regularity}

\subsection{Propagation of low regularity}

Recall that in Section 4 we have constructed a solution $(u, \rho)$ of (1.1) satisfying

$$
\begin{aligned}
& \frac{c_{0}}{2} \leq \rho(t, x) \leq 2 c_{0}^{-1}, \quad\|a\|_{\widetilde{L}_{T}^{\infty}\left(\dot{B}_{p, 1}^{\frac{3}{p}}\right)}+\|u\|_{\widetilde{L}_{T}^{\infty}\left(\dot{B}_{p, 1}^{\frac{3}{p}-1}\right)} \leq C_{0} E_{0}, \\
& \|a\|_{\widetilde{L}_{T}^{\infty}\left(\dot{B}_{p, 1}^{\frac{3}{p}}(\omega)\right)} \leq \eta_{1}, \quad\|u\|_{\widetilde{L}_{T}^{1}\left(\dot{B}_{p, 1}^{\frac{3}{p}+1}\right)}+\|u\|_{\widetilde{L}_{T}^{2}\left(\dot{B}_{p, 1}^{\frac{3}{p}}\right)} \leq \eta_{2},
\end{aligned}
$$

with $a(t, x)=(\rho(t, x)-\bar{\rho}) / \bar{\rho}, E_{0}=\left\|a_{0}\right\|_{\dot{B}_{p, 1}^{\frac{3}{p}}}+\left\|u_{0}\right\|_{\dot{B}_{p, 1}^{\frac{3}{p}-1}}$.

We will show that this solution allows to propagate the regularity of the initial data in Sobolev space with low regularity. This will ensure that one can use Hoff's theory for small energy initial data, since the norm of the initial data is small in Sobolev space with low regularity, but it is large in Sobolev space with high regularity.

Proposition 5.1 Let $p \in(3,6)$ and $1-\frac{3}{p}<\delta<\frac{3}{p}$. Assume that $(\rho, u)$ is a solution of (1.1) satisfying (5.1)). If $\left(a_{0}, u_{0}\right) \in \dot{H}^{1-\delta} \times \dot{H}^{-\delta}$, then

$$
\begin{aligned}
& \|a\|_{\widetilde{L}_{T}^{\infty}\left(\dot{B}_{2,2}^{1-\delta}\right)} \leq C\left(\left\|a_{0}\right\|_{\dot{H}^{1-\delta}}+\left\|u_{0}\right\|_{\dot{H}^{-\delta}}\right), \\
& \|u\|_{\widetilde{L}_{T}^{\infty}\left(\dot{B}_{2,2}^{-\delta}\right)} \leq C\left(1+E_{0}\right)\left(\left\|u_{0}\right\|_{\dot{H}^{-\delta}}+T\left\|a_{0}\right\|_{\dot{H}^{1-\delta}}\right), \\
& \|u\|_{\widetilde{L}_{T}^{1}\left(\dot{B}_{2,2}^{2-\delta}\right)}+\|u\|_{\widetilde{L}_{T}^{2}\left(\dot{B}_{2,2}^{1-\delta}\right)} \leq C\left(\left\|u_{0}\right\|_{\dot{H}^{-\delta}}+T\left\|a_{0}\right\|_{\dot{H}^{1-\delta}}\right) .
\end{aligned}
$$

Here the constant $C$ depends only on $\lambda, \mu, p, \delta, c_{0}$.

Proof. Set $\beta=1-\delta$. Without loss of generality, we can assume that $\eta_{1}, \eta_{2}, T$ small enough such that

$$
C \eta_{1} \leq \frac{1}{8}, \quad C \eta_{2} \leq \frac{1}{8}, \quad C T \leq \frac{1}{8}
$$

where $C$ is the constant appearing in the following estimates and depending on $\lambda, \mu, p, \delta, c_{0}$.

Step 1. Estimate for the transport equation

Apply the operator $\Delta_{j}$ to the first equation of (4.2) to obtain

$$
\partial_{t} \Delta_{j} a+u \cdot \nabla \Delta_{j} a=\Delta_{j} F+\left[u, \Delta_{j}\right] \cdot \nabla a,
$$

with $F=-(1+a) \operatorname{div} u$. Making $L^{2}$ energy estimate, we get

$$
\frac{1}{2} \frac{d}{d t}\left\|\Delta_{j} a\right\|_{2}^{2}-\frac{1}{2} \int_{\mathbf{R}^{3}}\left|\Delta_{j} a\right|^{2} \operatorname{div} u d x \leq\left(\left\|\Delta_{j} F\right\|_{2}+\left\|\left[u, \Delta_{j}\right] \cdot \nabla a\right\|_{2}\right)\left\|\Delta_{j} a\right\|_{2},
$$


this gives

$$
\left\|\Delta_{j} a(t)\right\|_{2} \leq\left\|\Delta_{j} a_{0}\right\|_{2}+\int_{0}^{t}\left(\left\|\Delta_{j} F\right\|_{2}+\left\|\left[u, \Delta_{j}\right] \cdot \nabla a\right\|_{2}+\frac{1}{2}\|\operatorname{div} u\|_{\infty}\left\|\Delta_{j} a\right\|_{2}\right) d \tau,
$$

from which, we deduce that

$$
\begin{aligned}
\|a\|_{\widetilde{L}_{t}^{\infty}\left(\dot{B}_{2,2}^{\beta}\right)} \leq & \left\|a_{0}\right\|_{\dot{B}_{2,2}^{\beta}}+C \int_{0}^{t}\|\operatorname{div} u\|_{\dot{B}_{p, 1}^{\frac{3}{p}}}\|a\|_{\tilde{L}_{\tau}^{\infty}\left(\dot{B}_{2,2}^{\beta}\right)} d \tau \\
& +\|F\|_{\widetilde{L}_{t}^{1}\left(\dot{B}_{2,2}^{\beta}\right)}+\left\|2^{j \beta}\right\|\left[u, \Delta_{j}\right] \cdot \nabla a\left\|_{L_{t}^{1}\left(L^{2}\right)}\right\|_{\ell^{2}} .
\end{aligned}
$$

We get by Lemma 5.5 and $\delta>1-\frac{3}{p}$ that

$$
\|F\|_{\widetilde{L}_{T}^{1}\left(\dot{B}_{2,2}^{\beta}\right)} \leq C\|u\|_{\widetilde{L}_{T}^{1}\left(\dot{B}_{2,2}^{\beta+1}\right)}\left(1+\|a\|_{\widetilde{L}_{T}^{\infty}\left(\dot{B}_{p, 1}^{\frac{3}{p}}\right)}\right) .
$$

and by Lemma 5.7

$$
\left\|2^{j \beta}\right\|\left[u, \Delta_{j}\right] \cdot \nabla a\left\|_{L_{T}^{1}\left(L^{2}\right)}\right\|_{\ell^{2}} \leq C\|u\|_{\tilde{L}_{T}^{1}\left(\dot{B}_{p, 1}^{\frac{3}{p}+1}\right)}\|a\|_{\tilde{L}_{T}^{\infty}\left(\dot{B}_{2,2}^{\beta}\right)} .
$$

Plugging them into (5.3) and using (5.1), we infer from Gronwall's inequality that

$$
\|a\|_{\widetilde{L}_{T}^{\infty}\left(\dot{B}_{2,2}^{\beta}\right)} \leq C\left(\left\|a_{0}\right\|_{\dot{H}^{1-\delta}}+\|u\|_{\widetilde{L}_{T}^{1}\left(\dot{B}_{2,2}^{\beta+1}\right)}\right) .
$$

Step 2. Estimate for the momentum equation

Apply the operator $\Delta_{j}$ to the second equation of (4.2) to obtain

$$
\partial_{t} \Delta_{j} u-\operatorname{div}\left(\bar{\mu} \nabla \Delta_{j} u\right)-\nabla\left(\bar{\lambda} \operatorname{div} \Delta_{j} u\right)=\Delta_{j} G+\operatorname{div}\left(\left[\Delta_{j}, \bar{\mu}\right] \nabla u\right)+\nabla\left(\left[\bar{\lambda}, \Delta_{j}\right] \operatorname{div} u\right),
$$

and recall that

$$
G=-u \cdot \nabla u-\frac{\bar{\rho} P^{\prime}(\rho)}{\rho} \nabla a+\frac{\mu}{\rho^{2}} \nabla \rho \cdot \nabla u+\frac{\lambda}{\rho^{2}} \nabla \rho \operatorname{div} u .
$$

Making $L^{2}$ energy estimate(see the proof of Proposition 3.3), we obtain

$$
\begin{aligned}
\|u\|_{\widetilde{L}_{T}^{\infty}\left(\dot{B}_{2,2}^{\beta-1}\right)} \leq & C\left(\left\|u_{0}\right\|_{\dot{B}_{2,2}^{\beta-1}}+\|G\|_{\widetilde{L}_{T}^{1}\left(\dot{B}_{2,2}^{\beta-1}\right)}\right) \\
& +C\left\|2^{j \beta}\left(\left\|\left[\Delta_{j}, \bar{\nu}\right] \nabla u\right\|_{L_{T}^{1}\left(L^{2}\right)}+\left\|\left[\Delta_{j}, \bar{\mu}\right] \nabla u\right\|_{L_{T}^{1}\left(L^{2}\right)}\right)\right\|_{\ell^{2}},
\end{aligned}
$$

and

$$
\begin{aligned}
& \|u\|_{\widetilde{L}_{T}^{1}\left(\dot{B}_{2,2}^{\beta+1}\right)}+\|u\|_{\widetilde{L}_{T}^{2}\left(\dot{B}_{2,2}^{\beta}\right)} \\
& \leq C\left(\left\|u_{0}\right\|_{\dot{B}_{2,2}^{\beta-1}(\omega)}+\|G\|_{\widetilde{L}_{T}^{1}\left(\dot{B}_{2,2}^{\beta-1}(\omega)\right)}\right) \\
& \quad+C\left\|2^{j \beta} w_{j}(T)\left(\left\|\left[\Delta_{j}, \bar{\nu}\right] \nabla u\right\|_{L_{T}^{1}\left(L^{2}\right)}+\left\|\left[\Delta_{j}, \bar{\mu}\right] \nabla u\right\|_{L_{T}^{1}\left(L^{2}\right)}\right)\right\|_{\ell^{2}} .
\end{aligned}
$$

First of all, by Lemma $5.6\left(\delta>1-\frac{3}{p}\right)$ and Lemma 2.8, we get

$$
\begin{aligned}
& \left\|2^{j \beta}\left(\left\|\left[\Delta_{j}, \bar{\nu}\right] \nabla u\right\|_{L_{T}^{1}\left(L^{2}\right)}+\left\|\left[\Delta_{j}, \bar{\mu}\right] \nabla u\right\|_{L_{T}^{1}\left(L^{2}\right)}\right)\right\|_{\ell^{2}} \\
& \leq C\|a\|_{\widetilde{L}_{T}^{\infty}\left(\dot{B}_{p, 1}^{\frac{3}{p}}\right)}\|u\|_{\widetilde{L}_{T}^{1}\left(\dot{B}_{2,2}^{\beta+1}\right)},
\end{aligned}
$$


and

$$
\begin{aligned}
& \left\|2^{j \beta} w_{j}(T)\left(\left\|\left[\Delta_{j}, \bar{\nu}\right] \nabla u\right\|_{L_{T}^{1}\left(L^{2}\right)}+\left\|\left[\Delta_{j}, \bar{\mu}\right] \nabla u\right\|_{L_{T}^{1}\left(L^{2}\right)}\right)\right\|_{\ell^{2}} \\
& \leq C\|a\|_{\widetilde{L}_{T}^{\infty}\left(\dot{B}_{p, 1}^{\frac{3}{p}}(\omega)\right)}\|u\|_{\widetilde{L}_{T}^{1}\left(\dot{B}_{2,2}^{\beta+1}\right)} .
\end{aligned}
$$

Applying Lemma 5.5 and Lemma 2.8 to $G$, we obtain

$$
\begin{gathered}
\|G\|_{\widetilde{L}_{T}^{1}\left(\dot{B}_{2,2}^{\beta-1}\right)} \leq C\left(\|u\|_{\widetilde{L}_{T}^{2}\left(\dot{B}_{p, 1}^{\frac{3}{p}}\right)}\|u\|_{\widetilde{L}_{T}^{2}\left(\dot{B}_{2,2}^{\beta}\right)}+\|a\|_{\widetilde{L}_{T}^{\infty}\left(\dot{B}_{p, 1}^{\frac{3}{p}}\right)}\|u\|_{\widetilde{L}_{T}^{1}\left(\dot{B}_{2,2}^{\beta+1}\right)}\right. \\
\left.+T\|a\|_{\widetilde{L}_{T}^{\infty}\left(\dot{B}_{2,2}^{\beta}\right)}\right),
\end{gathered}
$$

and

$$
\begin{gathered}
\|G\|_{\widetilde{L}_{T}^{1}\left(\dot{B}_{2,2}^{\beta-1}(\omega)\right)} \leq C\left(\|u\|_{\widetilde{L}_{T}^{2}\left(\dot{B}_{p, 1}^{\frac{3}{p}}\right)}\|u\|_{\widetilde{L}_{T}^{2}\left(\dot{B}_{2,2}^{\beta}\right)}+\|a\|_{\widetilde{L}_{T}^{\infty}\left(\dot{B}_{p, 1}^{\frac{3}{p}}(\omega)\right)}\|u\|_{\widetilde{L}_{T}^{1}\left(\dot{B}_{2,2}^{\beta+1}\right)}\right. \\
\left.+T\|a\|_{\widetilde{L}_{T}^{\infty}\left(\dot{B}_{2,2}^{\beta}\right)}\right) .
\end{gathered}
$$

Here we need $1-\frac{3}{p}<\delta<\frac{3}{p}$.

Plugging (5.7) and (5.9) into (5.5) and using (5.1), we get

$$
\|u\|_{\widetilde{L}_{T}^{\infty}\left(\dot{B}_{2,2}^{\beta-1}\right)} \leq C\left(\left\|u_{0}\right\|_{\dot{H}^{-\delta}}+T\|a\|_{\widetilde{L}_{T}^{\infty}\left(\dot{B}_{2,2}^{\beta}\right)}+E_{0}\|u\|_{\widetilde{L}_{T}^{1}\left(\dot{B}_{2,2}^{\beta+1}\right)}\right),
$$

and plugging (5.8) and (5.10) into (5.6), we have

$$
\|u\|_{\widetilde{L}_{T}^{1}\left(\dot{B}_{2,2}^{\beta+1}\right)}+\|u\|_{\widetilde{L}_{T}^{2}\left(\dot{B}_{2,2}^{\beta}\right)} \leq C\left(\left\|u_{0}\right\|_{\dot{H}^{-\delta}}+T\|a\|_{\widetilde{L}_{T}^{\infty}\left(\dot{B}_{2,2}^{\beta}\right)}\right)
$$

Then Proposition 5.1 follows by combining (5.11)-(5.12) with (5.4).

\subsection{Propagation of high regularity}

We will show that the solution constructed in Section 4 can also propagate the regularity of the initial data in Sobolev space with high regularity.

Proposition 5.2 Assume that $(\rho, u)$ is a solution of (1.1) on $[0, T]$, which satisfies

$$
\rho \geq c_{0}, \quad a \in \widetilde{L}^{\infty}\left(0, T ; \dot{B}_{p, 1}^{\frac{3}{p}}\right), \quad u \in \widetilde{L}^{\infty}\left(0, T ; \dot{B}_{p, 1}^{\frac{3}{p}-1}\right) \cap \widetilde{L}^{1}\left(0, T ; \dot{B}_{p, 1}^{\frac{3}{p}+1}\right) .
$$

If $\left(a_{0}, u_{0}\right) \in H^{s} \times H^{s-1}$ for $s \geq 3$, then we have

$$
a \in \widetilde{L}^{\infty}\left(0, T ; \dot{B}_{2,2}^{s}\right), \quad u \in \widetilde{L}^{\infty}\left(0, T ; \dot{B}_{2,2}^{s-1}\right) \cap \widetilde{L}^{1}\left(0, T ; \dot{B}_{2,2}^{s+1}\right) .
$$

Proof. Due to (5.13), we can divide the time interval $[0, T]$ into finite many small intervals $\left[T_{i}, T_{i+1}\right]$ with $i=0, \cdots, N$ such that

$$
\begin{aligned}
& \|a\|_{\widetilde{L}^{\infty}\left(T_{i}, T_{i+1} ; \dot{B}_{p, 1}^{\frac{3}{p}}\left(\omega^{i}\right)\right)} \leq \epsilon, \\
& \|u\|_{\widetilde{L}^{1}\left(T_{i}, T_{i+1} ; \dot{B}_{p, 1}^{\frac{3}{p}+1}\right)}+\|u\|_{\widetilde{L}^{2}\left(T_{i}, T_{i+1} ; \dot{B}_{p, 1}^{\frac{3}{p}}\right)} \leq \epsilon,
\end{aligned}
$$


for some $\epsilon$ small enough, see Remark 5.3. Here the weight on each $\left[T_{i}, T_{i+1}\right]$ is given by

$$
\omega_{k}^{i}=\sum_{\ell \geq k} 2^{k-\ell} e_{\ell}^{i}, \quad e_{\ell}^{i}=\left(1-e^{-c 2^{2 \ell}\left(T_{i+1}-T_{i}\right)}\right)^{\frac{1}{2}} .
$$

First of all, we also have(see 5.3)

$$
\begin{aligned}
\|a\|_{\widetilde{L}_{t}^{\infty}\left(\dot{B}_{2,2}^{s}\right)} \leq & \left\|a_{0}\right\|_{\dot{B}_{2,2}^{s}}+C \int_{0}^{t}\|\operatorname{div} u\|_{\dot{B}_{p, 1}^{\frac{3}{p}}}\|a\|_{\tilde{L}_{\tau}^{\infty}\left(\dot{B}_{2,2}^{s}\right)} d \tau \\
& +\|F\|_{\widetilde{L}_{t}^{1}\left(\dot{B}_{2,2}^{s}\right)}+\left\|2^{j s}\right\|\left[u, \Delta_{j}\right] \cdot \nabla a\left\|_{L_{t}^{1}\left(L^{2}\right)}\right\|_{\ell^{2}} .
\end{aligned}
$$

By Lemma 5.8, we have

$$
\|F\|_{\widetilde{L}_{t}^{1}\left(\dot{B}_{2,2}^{s}\right)} \leq C\left(\|u\|_{\widetilde{L}_{t}^{1}\left(\dot{B}_{2,2}^{s+1}\right)}+\|a\|_{\widetilde{L}_{t}^{\infty}\left(\dot{B}_{p, 1}^{\frac{3}{p}}\right)}\|u\|_{\widetilde{L}_{t}^{1}\left(\dot{B}_{2,2}^{s+1}\right)}+\|a\|_{\widetilde{L}_{t}^{\infty}\left(\dot{B}_{2,2}^{s}\right)}\|u\|_{\widetilde{L}_{t}^{1}\left(\dot{B}_{p, 1}^{\frac{3}{p}+1}\right)}\right),
$$

and by Lemma 5.10 .

$$
\left\|2^{j s}\right\|\left[u, \Delta_{j}\right] \cdot \nabla a\left\|_{L_{t}^{1}\left(L^{2}\right)}\right\|_{\ell^{2}} \leq C\left(\|u\|_{\tilde{L}_{t}^{1}\left(\dot{B}_{p, 1}^{\frac{3}{p}+1}\right)}\|a\|_{\tilde{L}_{t}^{\infty}\left(\dot{B}_{2,2}^{s}\right)}+\|u\|_{\tilde{L}_{t}^{1}\left(\dot{B}_{2,2}^{s+1}\right)}\|a\|_{\tilde{L}_{t}^{\infty}\left(\dot{B}_{p, 1}^{\frac{3}{p}}\right)}\right),
$$

from which and (5.14), we get by Gronwall's inequality that for $t \in\left[0, T_{1}\right]$,

$$
\|a\|_{\widetilde{L}_{t}^{\infty}\left(\dot{B}_{2,2}^{s}\right)} \leq C\left(\left\|a_{0}\right\|_{\dot{B}_{2,2}^{s}}+\|u\|_{\widetilde{L}_{t}^{1}\left(\dot{B}_{2,2}^{s+1}\right)}\right) .
$$

Making $H^{s-1}$ energy estimate for the momentum equation, we get(see [5.5 and (5.6) )

$$
\begin{aligned}
\|u\|_{\tilde{L}_{t}^{\infty}\left(\dot{B}_{2,2}^{s-1}\right)} \leq & C\left(\left\|u_{0}\right\|_{\dot{B}_{2,2}^{s-1}}+\|G\|_{\widetilde{L}_{t}^{1}\left(\dot{B}_{2,2}^{s-1}\right)}\right) \\
& +C\left\|2^{j s}\left(\left\|\left[\Delta_{j}, \bar{\nu}\right] \nabla u\right\|_{L_{t}^{1}\left(L^{2}\right)}+\left\|\left[\Delta_{j}, \bar{\mu}\right] \nabla u\right\|_{L_{t}^{1}\left(L^{2}\right)}\right)\right\|_{\ell^{2}},
\end{aligned}
$$

and

$$
\begin{aligned}
& \|u\|_{\widetilde{L}_{t}^{1}\left(\dot{B}_{2,2}^{s+1}\right)}+\|u\|_{\widetilde{L}_{t}^{2}\left(\dot{B}_{2,2}^{s}\right)} \\
& \leq C\left(\left\|u_{0}\right\|_{\dot{B}_{2,2}^{s-1}}+\|G\|_{\widetilde{L}_{t}^{1}\left(\dot{B}_{2,2}^{s-1}(\omega)\right)}\right) \\
& \quad+C\left\|2^{j s} w_{j}(t)\left(\left\|\left[\Delta_{j}, \bar{\nu}\right] \nabla u\right\|_{L_{t}^{1}\left(L^{2}\right)}+\left\|\left[\Delta_{j}, \bar{\mu}\right] \nabla u\right\|_{L_{t}^{1}\left(L^{2}\right)}\right)\right\|_{\ell^{2}} .
\end{aligned}
$$

By Lemma 5.8 and Lemma 2.8, we have

$$
\begin{aligned}
& \|G\|_{\widetilde{L}_{t}^{1}\left(\dot{B}_{2,2}^{s-1}(\omega)\right)} \leq C\left(\|u\|_{\widetilde{L}_{t}^{2}\left(\dot{B}_{p, 1}^{\frac{3}{p}}\right)}\|u\|_{\widetilde{L}_{t}^{2}\left(\dot{B}_{2,2}^{s}\right)}+\|u\|_{\widetilde{L}_{t}^{2}\left(\dot{B}_{p, 1}^{\frac{3}{p}}\right)}\|u\|_{\widetilde{L}_{T}^{2}\left(\dot{B}_{2,2}^{s}\right)}\right. \\
& \left.\quad+\|a\|_{\widetilde{L}_{t}^{\infty}\left(\dot{B}_{p, 1}^{\frac{3}{p}}(\omega)\right)}\|u\|_{\widetilde{L}_{t}^{1}\left(\dot{B}_{2,2}^{s+1}\right)}+\|a\|_{\widetilde{L}_{t}^{\infty}\left(\dot{B}_{2,2}^{s}\right)}\|u\|_{\widetilde{L}_{t}^{1}\left(\dot{B}_{p, 1}^{\frac{3}{p}+1}\right)}+t\|a\|_{\widetilde{L}_{t}^{\infty}\left(\dot{B}_{2,2}^{s}\right)}\right),
\end{aligned}
$$

and Lemma 5.9 and Lemma 2.8 ,

$$
\begin{aligned}
& \left\|2^{j s} w_{j}(t)\left(\left\|\left[\Delta_{j}, \bar{\nu}\right] \nabla u\right\|_{L_{t}^{1}\left(L^{2}\right)}+\left\|\left[\Delta_{j}, \bar{\mu}\right] \nabla u\right\|_{L_{t}^{1}\left(L^{2}\right)}\right)\right\|_{\ell^{2}} \\
& \quad \leq C\left(\|a\|_{\tilde{L}_{t}^{\infty}\left(\dot{B}_{p, 1}^{\frac{3}{p}}(\omega)\right)}\|u\|_{\tilde{L}_{t}^{1}\left(\dot{B}_{2,2}^{s+1}\right)}+\|a\|_{\tilde{L}_{t}^{\infty}\left(\dot{B}_{2,2}^{s}\right)}\|u\|_{\tilde{L}_{t}^{1}\left(\dot{B}_{p, 1}^{\frac{3}{p}+1}\right)}\right)
\end{aligned}
$$


which along with (5.17) gives that

$$
\|u\|_{\widetilde{L}_{t}^{1}\left(\dot{B}_{2,2}^{s+1}\right)}+\|u\|_{\widetilde{L}_{t}^{2}\left(\dot{B}_{2,2}^{s}\right)} \leq C\left(\left\|u_{0}\right\|_{\dot{H}^{s-1}}+\|a\|_{\widetilde{L}_{t}^{\infty}\left(\dot{B}_{2,2}^{s}\right)}\left(\|u\|_{\widetilde{L}_{t}^{1}\left(\dot{B}_{p, 1}^{\frac{3}{p}+1}\right)}+t\right)\right),
$$

from which and (5.15), it follows that for any $t \in\left[0, T_{1}\right]$,

$$
\|a\|_{\widetilde{L}_{t}^{\infty}\left(\dot{B}_{2,2}^{s}\right)}+\|u\|_{\widetilde{L}_{t}^{1}\left(\dot{B}_{2,2}^{s+1}\right)}+\|u\|_{\widetilde{L}_{t}^{2}\left(\dot{B}_{2,2}^{s}\right)} \leq C\left(\left\|a_{0}\right\|_{\dot{H}^{s}}+\left\|u_{0}\right\|_{\dot{H}^{s-1}}\right) .
$$

On the other hand, by Lemma 5.8 and Lemma 2.8 again, we have

$$
\begin{aligned}
& \|G\|_{\widetilde{L}_{t}^{1}\left(\dot{B}_{2,2}^{s-1}\right)} \leq C\left(\|u\|_{\widetilde{L}_{t}^{2}\left(\dot{B}_{p, 1}^{\frac{3}{p}}\right)}\|u\|_{\widetilde{L}_{t}^{2}\left(\dot{B}_{2,2}^{s}\right)}+\|u\|_{\widetilde{L}_{t}^{2}\left(\dot{B}_{p, 1}^{\frac{3}{p}}\right)}\|u\|_{\widetilde{L}_{T}^{2}\left(\dot{B}_{2,2}^{s}\right)}\right. \\
& \left.\quad+\|a\|_{\widetilde{L}_{t}^{\infty}\left(\dot{B}_{p, 1}^{\frac{3}{p}}\right)}\|u\|_{\widetilde{L}_{t}^{1}\left(\dot{B}_{2,2}^{s+1}\right)}+\|a\|_{\widetilde{L}_{t}^{\infty}\left(\dot{B}_{2,2}^{s}\right)}\|u\|_{\widetilde{L}_{t}^{1}\left(\dot{B}_{p, 1}^{\frac{3}{p}+1}\right)}+t\|a\|_{\widetilde{L}_{t}^{\infty}\left(\dot{B}_{2,2}^{s}\right)}\right),
\end{aligned}
$$

and by Lemma 5.9 and Lemma 2.8 ,

$$
\begin{aligned}
& \left\|2^{j s}\left(\left\|\left[\Delta_{j}, \bar{\nu}\right] \nabla u\right\|_{L_{t}^{1}\left(L^{2}\right)}+\left\|\left[\Delta_{j}, \bar{\mu}\right] \nabla u\right\|_{L_{t}^{1}\left(L^{2}\right)}\right)\right\|_{\ell^{2}} \\
& \quad \leq C\left(\|a\|_{\tilde{L}_{t}^{\infty}\left(\dot{B}_{p, 1}^{\frac{3}{p}}\right)}\|u\|_{\tilde{L}_{t}^{1}\left(\dot{B}_{2,2}^{s+1}\right)}+\|a\|_{\tilde{L}_{t}^{\infty}\left(\dot{B}_{2,2}^{s}\right)}\|u\|_{\tilde{L}_{t}^{1}\left(\dot{B}_{p, 1}^{\frac{3}{p}+1}\right)}\right),
\end{aligned}
$$

which along with (5.16) and (5.15) implies that for any $t \in\left[0, T_{1}\right]$,

$$
\|u\|_{\widetilde{L}_{t}^{\infty}\left(\dot{B}_{2,2}^{s-1}\right)} \leq C\left(1+\|a\|_{\tilde{L}_{t}^{\infty}\left(\dot{B}_{p, 1}^{\frac{3}{p}}\right)}\right)\left(\left\|a_{0}\right\|_{\dot{H}^{s}}+\left\|u_{0}\right\|_{\dot{H}^{s-1}}\right) .
$$

Then the proposition follows by repeating the above process in each interval $\left[T_{i}, T_{i+1}\right]$.

Remark 5.3 Thanks to $a \in \widetilde{L}^{\infty}\left(0, T ; \dot{B}_{p, 1}^{\frac{3}{p}}\right)$, one can choose $N_{1}$ big enough so that

$$
\sum_{|j|>N_{1}} 2^{j \frac{3}{p}}\left\|\Delta_{j} a(t)\right\|_{L^{\infty}\left(0, T ; L^{p}\right)} \leq \frac{\epsilon}{4}
$$

Fix $N_{1}$, one can divide $[0, T]$ into finite many small intervals $\left[T_{i}, T_{i+1}\right]$ with $i=0, \cdots, N$ such that for $i=0, \cdots, N$

$$
\sum_{|j| \leq N_{1}} 2^{j \frac{3}{p}} \omega_{j}^{i}\left\|\Delta_{j} a(t)\right\|_{L^{\infty}\left(T_{i}, T_{i+1} ; L^{p}\right)} \leq \frac{\epsilon}{2} .
$$

Then we conclude that

$$
\begin{aligned}
& \|a\|_{\widetilde{L}^{\infty}\left(T_{i}, T_{i+1} ; \dot{B}_{p, 1}^{\frac{3}{p}}\left(\omega^{i}\right)\right)} \\
& \leq 2 \sum_{|j|>N_{1}} 2^{j \frac{3}{p}}\left\|\Delta_{j} a(t)\right\|_{L^{\infty}\left(T_{i}, T_{i+1} ; L^{p}\right)}+\sum_{|j| \leq N_{1}} 2^{j \frac{3}{p}} \omega_{j}^{i}\left\|\Delta_{j} a(t)\right\|_{L^{\infty}\left(T_{i}, T_{i+1} ; L^{p}\right)} \leq \epsilon .
\end{aligned}
$$




\subsection{Some technical lemmas}

Lemma 5.4 Let $p \geq 2,1 \leq q_{1}, q_{2}, q \leq \infty$ with $\frac{1}{q_{1}}+\frac{1}{q_{2}}=\frac{1}{q}$. Then there hold (a) if $s_{2}<\frac{3}{p}$, we have

$$
\left\|T_{g} f\right\|_{\widetilde{L}_{T}^{q}\left(\dot{B}_{2,2}^{s_{1}+s_{2}-\frac{3}{p}}(\omega)\right)} \leq C\|f\|_{\widetilde{L}_{T}^{q_{1}}\left(\dot{B}_{p, 1}^{s_{1}}(\omega)\right)}\|g\|_{\widetilde{L}_{T}^{q_{2}}\left(\dot{B}_{2,2}^{s_{2}}\right)} ;
$$

(b) if $s_{1} \leq \frac{3}{p}-1$, we have

$$
\left\|T_{f} g\right\|_{\widetilde{L}_{T}^{q}\left(\dot{B}_{2,2}^{s_{1}+s_{2}-\frac{3}{p}}(\omega)\right)} \leq C\|f\|_{\widetilde{L}_{T}^{q_{1}}\left(\dot{B}_{p, 1}^{s_{1}}(\omega)\right)}\|g\|_{\widetilde{L}_{T}^{q_{2}}\left(\dot{B}_{2,2}^{s_{2}}\right)} ;
$$

(c) if $s_{1}+s_{2}>0$, we have

$$
\|R(f, g)\|_{\widetilde{L}_{T}^{q}\left(\dot{B}_{2,2}^{s_{1}+s_{2}-\frac{3}{p}}(\omega)\right)} \leq C\|f\|_{\widetilde{L}_{T}^{q_{1}}\left(\dot{B}_{p, 1}^{s_{1}}(\omega)\right)}\|g\|_{\widetilde{L}_{T}^{q_{2}}\left(\dot{B}_{2,2}^{s_{2}}\right)} .
$$

Proof. We first prove (a). Due to (2.1), we have

$$
\Delta_{j}\left(T_{g} f\right)=\sum_{\left|j^{\prime}-j\right| \leq 4} \Delta_{j}\left(S_{j^{\prime}-1} g \Delta_{j^{\prime}} f\right),
$$

then we get by Lemma 2.1 and (2.4) that

$$
\begin{aligned}
\left\|T_{g} f\right\|_{\widetilde{L}_{T}^{q}\left(\dot{B}_{2,2}^{s_{1}+s_{2}-\frac{3}{p}}(\omega)\right)} & =\left\|2^{j\left(s_{1}+s_{2}-\frac{3}{p}\right)} \omega_{j}(T)\right\| \Delta_{j}\left(T_{g} f\right)\left\|_{L_{T}^{q}\left(L^{2}\right)}\right\|_{\ell^{2}} \\
& \leq C\left\|2^{j\left(s_{1}+s_{2}-\frac{3}{p}\right)} \omega_{j}(T)\right\| S_{j-1} g\left\|_{L_{T}^{q_{2}}\left(L^{\frac{2 p}{p-2}}\right)}\right\| \Delta_{j} f\left\|_{L_{T}^{q_{1}}\left(L^{p}\right)}\right\|_{\ell^{2}} \\
& \leq C\|f\|_{\widetilde{L}_{T}^{q_{1}\left(\dot{B}_{p, 1}^{s_{1}}(\omega)\right)}\|g\|_{\tilde{L}_{T}^{q_{2}}\left(\dot{B}_{2,2}^{s_{2}}\right)}}
\end{aligned}
$$

where we used in the last inequality

$$
\left\|S_{j-1} g\right\|_{L_{T}^{q_{2}}\left(L^{\frac{2 p}{p-2}}\right)} \leq C \sum_{\ell \leq j-2} 2^{\ell \frac{3}{p}}\left\|\Delta_{\ell} g\right\|_{L_{T}^{q_{2}}\left(L^{2}\right)} \leq C 2^{j\left(-s_{2}+\frac{3}{p}\right)}\|g\|_{\tilde{L}_{T}^{q_{2}}\left(\dot{B}_{2,2}^{s_{2}}\right)} .
$$

We next prove (b). Similarly, we have

$$
\begin{aligned}
\left\|T_{f} g\right\|_{\widetilde{L}_{T}^{q}\left(\dot{B}_{2,2}^{s_{1}+s_{2}-\frac{3}{p}}(\omega)\right)} & =\left\|2^{j\left(s_{1}+s_{2}-\frac{3}{p}\right)} \omega_{j}(T)\right\| \Delta_{j}\left(T_{f} g\right)\left\|_{L_{T}^{q}\left(L^{2}\right)}\right\|_{\ell^{2}} \\
& \leq C\left\|2^{j\left(s_{1}+s_{2}-\frac{3}{p}\right)} \omega_{j}(T)\right\| S_{j-1} f\left\|_{L_{T}^{q_{1}}\left(L^{\infty}\right)}\right\| \Delta_{j} g\left\|_{L_{T}^{q_{2}}\left(L^{2}\right)}\right\|_{\ell^{2}},
\end{aligned}
$$

and by Lemma 2.1 and (2.4), we have

$$
\begin{aligned}
\omega_{j}(T)\left\|S_{j-1} f\right\|_{L_{T}^{q_{1}}\left(L^{\infty}\right)} & \leq C 2^{j} \sum_{\ell \leq j-2} 2^{\ell\left(\frac{3}{p}-1\right)} \omega_{\ell}(T)\left\|\Delta_{\ell} f\right\|_{L_{T}^{q_{1}}\left(L^{p}\right)} \\
& \leq C 2^{j\left(\frac{3}{p}-s_{1}\right)} \sum_{\ell \leq j-2} 2^{\ell s_{1}} \omega_{\ell}(T)\left\|\Delta_{\ell} f\right\|_{L_{T}^{q_{1}}\left(L^{p}\right)} \\
& \leq C 2^{j\left(\frac{3}{p}-s_{1}\right)}\|f\|_{\widetilde{L}_{T}^{q_{1}}\left(\dot{B}_{p, 1}^{s_{1}}(\omega)\right)}
\end{aligned}
$$


which lead to (b). Finally, let us prove (c). Noticing that

$$
\Delta_{j}(R(f, g))=\sum_{j^{\prime} \geq j-3} \Delta_{j}\left(\Delta_{j^{\prime}} f \widetilde{\Delta}_{j^{\prime}} g\right)
$$

then we get by Lemma 2.1 that

$$
\begin{aligned}
& \|R(f, g)\|_{\widetilde{L}_{T}^{q}\left(\dot{B}_{2,2}^{s_{1}+s_{2}-\frac{3}{p}}(\omega)\right)} \\
& \leq C\left\|\sum_{j^{\prime} \geq j-3} 2^{j\left(s_{1}+s_{2}\right)} \omega_{j}(T)\right\| \Delta_{j^{\prime}} f\left\|_{L_{T}^{q_{1}}\left(L^{p}\right)}\right\| \widetilde{\Delta}_{j^{\prime}} g\left\|_{L_{T}^{q_{2}\left(L^{2}\right)}}\right\|_{\ell^{2}} \\
& \leq C\left\|\sum_{j^{\prime} \geq j-3} \sum_{\ell \geq j} 2^{j-\ell} e_{\ell}(T) 2^{j\left(s_{1}+s_{2}\right)}\right\| \Delta_{j^{\prime}} f\left\|_{L_{T}^{q_{1}}\left(L^{p}\right)}\right\| \widetilde{\Delta}_{j^{\prime}} g\left\|_{\left.L_{T}^{q_{2}\left(L^{2}\right)}\right)}\right\|_{\ell^{2}} \\
& =\left\|\sum_{j^{\prime} \geq j-3} \sum_{\ell \geq j, j^{\prime}} \cdots\right\|_{\ell^{2}}+\left\|\sum_{j^{\prime} \geq j-3} \sum_{j^{\prime} \geq \ell \geq j} \cdots\right\|_{\ell^{2}} \\
& =I+I I .
\end{aligned}
$$

In the following, we denote $\left\{c_{j}\right\}$ by a sequence in $\ell^{2}$. Noting that

$$
\sum_{\ell \geq j, j^{\prime}} 2^{j-\ell} e_{\ell}(T) \leq 2^{j-j^{\prime}} \sum_{\ell \geq j^{\prime}} 2^{j^{\prime}-\ell} e_{\ell}(T)=2^{j-j^{\prime}} w_{j^{\prime}}(T)
$$

we infer that

$$
\begin{aligned}
I & \leq C\left\|\sum_{j^{\prime} \geq j-3} \omega_{j^{\prime}}(T) 2^{j\left(s_{1}+s_{2}\right)} 2^{j-j^{\prime}}\right\| \Delta_{j^{\prime}} f\left\|_{L_{T}^{q_{1}}\left(L^{p}\right)}\right\| \widetilde{\Delta}_{j^{\prime}} g\left\|_{L_{T}^{q_{2}}\left(L^{2}\right)}\right\|_{\ell^{2}} \\
& \leq C\left\|\sum_{j^{\prime} \geq j-3} \omega_{j^{\prime}}(T) 2^{\left(j-j^{\prime}\right)\left(s_{1}+s_{2}+1\right)} 2^{j^{\prime} s_{1}} c_{j^{\prime}}\right\| \Delta_{j^{\prime}} f\left\|_{L_{T}^{q_{1}}\left(L^{p}\right)}\right\|_{\ell^{2}}\|g\|_{\widetilde{L}_{T}^{q_{2}}\left(\dot{B}_{2,2}^{s_{2}}\right)} \\
& \leq C\|f\|_{\widetilde{L}_{T}^{q_{1}}\left(\dot{B}_{p, 1}^{\left.s_{1}(\omega)\right)}\right)}\|g\|_{\widetilde{L}_{T}^{q_{2}\left(\dot{B}_{2,2}^{s_{2}}\right)}} .
\end{aligned}
$$

and thanks to $e_{\ell}(T) \leq e_{j^{\prime}}(T) \leq \omega_{j^{\prime}}(T)$ for $\ell \leq j^{\prime}$, we get

$$
\begin{aligned}
I I & \leq C\left\|\sum_{j^{\prime} \geq j-3} \omega_{j^{\prime}}(T) 2^{j\left(s_{1}+s_{2}\right)}\right\| \Delta_{j^{\prime}} f\left\|_{L_{T}^{q_{1}}\left(L^{p}\right)}\right\| \widetilde{\Delta}_{j^{\prime}} g\left\|_{L_{T}^{q_{2}}\left(L^{2}\right)}\right\|_{\ell^{2}} \\
& \leq C\left\|\sum_{j^{\prime} \geq j-3} \omega_{j^{\prime}}(T) 2^{\left(j-j^{\prime}\right)\left(s_{1}+s_{2}\right)} 2^{j^{\prime} s_{1}} c_{j^{\prime}}\right\| \Delta_{j^{\prime}} f\left\|_{p}\right\|_{\ell^{2}}\|g\|_{\widetilde{L}_{T}^{q_{2}}\left(\dot{B}_{2,2}^{s_{2}}\right)} \\
& \leq C\|f\|_{\widetilde{L}_{T}^{q_{1}}\left(\dot{B}_{p, 1}^{\left.s_{1}(\omega)\right)}\right.}\|g\|_{\widetilde{L}_{T}^{q_{2}}\left(\dot{B}_{2,2}^{s_{2}}\right)},
\end{aligned}
$$

This proves (c).

From Lemma 5.4 and its proof, it is easy to see that

Lemma 5.5 Let $p \geq 2, s_{1} \leq \frac{3}{p}, s_{2}<\frac{3}{p}, s_{1}+s_{2}>0$, and $1 \leq q, q_{1}, q_{2} \leq \infty$ with $\frac{1}{q_{1}}+\frac{1}{q_{2}}=\frac{1}{q}$. Then we have

$$
\|f g\|_{\widetilde{L}_{T}^{q}\left(\dot{B}_{2,2}^{\left.s_{1}+s_{2}-\frac{3}{p}\right)}\right.} \leq C\|f\|_{\widetilde{L}_{T}^{q_{1}}\left(\dot{B}_{p, 1}^{s_{1}}\right)}\|g\|_{\widetilde{L}_{T}^{q_{2}}\left(\dot{B}_{2,2}^{s_{2}}\right)}
$$

and if $s_{1} \leq \frac{3}{p}-1$, then

$$
\|f g\|_{\widetilde{L}_{T}^{q}\left(\dot{B}_{2,2}^{s_{1}+s_{2}-\frac{3}{p}}(\omega)\right)} \leq C\|f\|_{\widetilde{L}_{T}^{q_{1}}\left(\dot{B}_{p, 1}^{s_{1}}(\omega)\right)}\|g\|_{\widetilde{L}_{T}^{q_{2}}\left(\dot{B}_{2,2}^{s_{2}}\right)}
$$


Lemma 5.6 Let $p \geq 2$ and $s \in\left(-\frac{3}{p}, \frac{3}{p}\right)$. Then there holds

$$
\begin{aligned}
& \left\|2^{j s}\right\|\left[\Delta_{j}, f\right] \nabla g\left\|_{L_{T}^{1}\left(L^{2}\right)}\right\|_{\ell^{2}} \leq C\|f\|_{\tilde{L}_{T}^{\infty}\left(\dot{B}_{p, 1}^{\frac{3}{p}}\right)}\|g\|_{\tilde{L}_{T}^{1}\left(\dot{B}_{2,2}^{s+1}\right)}, \\
& \left\|2^{j s} \omega_{j}(T)\right\|\left[\Delta_{j}, f\right] \nabla g\left\|_{L_{T}^{1}\left(L^{2}\right)}\right\|_{\ell^{2}} \leq C\|f\|_{\tilde{L}_{T}^{\infty}\left(\dot{B}_{p, 1}^{\frac{3}{p}}(\omega)\right)}\|g\|_{\tilde{L}_{T}^{1}\left(\dot{B}_{2,2}^{s+1}\right)} .
\end{aligned}
$$

Proof. The proof is almost the same as Lemma 2.9. We write

$$
\left[f, \Delta_{j}\right] \nabla g=\left[T_{f}, \Delta_{j}\right] \nabla g+T_{\Delta_{j} \nabla g} f+R\left(f, \Delta_{j} \nabla g\right)-\Delta_{j}\left(T_{\nabla g} f\right)-\Delta_{j} R(f, \nabla g) .
$$

It follows from Lemma 5.4 (a), (c) that

$$
\begin{aligned}
& \left\|2^{j s} \omega_{j}(T)\right\| \Delta_{j}\left(T_{\nabla g} f\right)\left\|_{L_{T}^{1}\left(L^{2}\right)}\right\|_{\ell^{2}} \leq C\|f\|_{\tilde{L}_{T}^{\infty}\left(\dot{B}_{p, 1}^{\frac{3}{p}}(\omega)\right)}\|g\|_{\tilde{L}_{T}^{1}\left(\dot{B}_{2,2}^{s+1}\right)}, \\
& \left\|2^{j s} \omega_{j}(T)\right\| \Delta_{j} R(f, \nabla g)\left\|_{L_{T}^{1}\left(L^{2}\right)}\right\|_{\ell^{2}} \leq C\|f\|_{\tilde{L}_{T}^{\infty}\left(\dot{B}_{p, 1}^{\frac{3}{p}}(\omega)\right)}\|g\|_{\tilde{L}_{T}^{1}\left(\dot{B}_{2,2}^{s+1}\right)} .
\end{aligned}
$$

Recalling (2.5) and by Lemma 2.1 and (2.4), we get

$$
\begin{aligned}
& \left\|\omega_{j}(T) 2^{j s}\right\| T_{\Delta_{j} \nabla g}^{\prime} f\left\|_{L_{T}^{1}\left(L^{2}\right)}\right\|_{\ell^{2}} \\
& \leq C\left\|\omega_{j}(T) 2^{j(s+1)}\right\| \Delta_{j} g\left\|_{L_{T}^{1}\left(L^{2}\right)} \sum_{j^{\prime} \geq j-2}\right\| \Delta_{j^{\prime}} f\left\|_{L_{T}^{\infty}\left(L^{\infty}\right)}\right\|_{\ell^{2}} \\
& \leq C\left\|2^{j(s+1)}\right\| \Delta_{j} g\left\|_{L_{T}^{1}\left(L^{2}\right)} \sum_{j^{\prime} \geq j-2} \omega_{j^{\prime}}(T) 2^{j^{\prime} \frac{3}{p}}\right\| \Delta_{j^{\prime}} f\left\|_{L_{T}^{\infty}\left(L^{p}\right)}\right\|_{\ell^{2}} \\
& \leq C\|f\|_{\widetilde{L}_{T}^{\infty}\left(\dot{B}_{p, 1}^{\frac{3}{p}}(\omega)\right)}\|g\|_{\widetilde{L}_{T}^{1}\left(\dot{B}_{2,2}^{s+1}\right)} .
\end{aligned}
$$

Note that

$$
\begin{gathered}
{\left[T_{f}, \Delta_{j}\right] \partial_{k} g=\sum_{\left|j^{\prime}-j\right| \leq 4} 2^{4 j} \int_{\mathbf{R}^{3}} \int_{0}^{1} y \cdot \nabla S_{j^{\prime}-1} f(x-\tau y) d \tau \partial_{k} h\left(2^{j} y\right) \Delta_{j^{\prime}} g(x-y) d y} \\
+2^{3 j} \int_{\mathbf{R}^{3}} h\left(2^{j}(x-y)\right) \partial_{k} S_{j^{\prime}-1} f(y) \Delta_{j^{\prime}} g(y) d y
\end{gathered}
$$

from which and Young's inequality, we infer that

$$
\left\|\omega_{j}(T) 2^{j s}\right\|\left[T_{f}, \Delta_{j}\right] \nabla g\left\|_{L_{T}^{1}\left(L^{2}\right)}\right\|_{\ell^{2}} \leq C\|f\|_{\widetilde{L}_{T}^{\infty}\left(\dot{B}_{p, 1}^{\frac{3}{p}}(\omega)\right)}\|g\|_{\widetilde{L}_{T}^{1}\left(\dot{B}_{2,2}^{s+1}\right)} .
$$

Summing up the above estimates yield the second inequality, the first one is similar.

From the proof of Lemma [5.6, it is easy to see that

Lemma 5.7 Let $p \geq 2$ and $s \in\left(-\frac{3}{p}, \frac{3}{p}+1\right)$. Then there holds

$$
\left\|2^{j s}\right\|\left[\Delta_{j}, f\right] \nabla g\left\|_{L_{T}^{1}\left(L^{2}\right)}\right\|_{\ell^{2}} \leq C\|f\|_{\tilde{L}_{T}^{\infty}\left(\dot{B}_{p, 1}^{\frac{3}{p}+1}\right)}\|g\|_{\tilde{L}_{T}^{1}\left(\dot{B}_{2,2}^{s}\right)} .
$$

The following lemmas are used in the proof of propagation of high regularity. Since the proof is very similar to Lemma 5.5.Lemma 5.7, we state them without proof. 
Lemma 5.8 Let $p \geq 2, s_{1}, s_{1}^{\prime} \leq \frac{3}{p}, s_{1}+s_{2}=s_{1}^{\prime}+s_{2}^{\prime}>0$, and $1 \leq q, q_{1}, q_{2} \leq \infty$ with $\frac{1}{q_{1}}+\frac{1}{q_{2}}=\frac{1}{q}$. Then there holds

$$
\|f g\|_{\widetilde{L}_{T}^{q}\left(\dot{B}_{2,2}^{\left.s_{1}+s_{2}-\frac{3}{p}\right)}\right.} \leq C\left(\|f\|_{\widetilde{L}_{T}^{q_{1}}\left(\dot{B}_{p, 1}^{s_{1}}\right)}\|g\|_{\widetilde{L}_{T}^{q_{2}}\left(\dot{B}_{2,2}^{s_{2}}\right)}+\|f\|_{\widetilde{L}_{T}^{q_{1}\left(\dot{B}_{2,2}^{s_{2}^{\prime}}\right)}}\|g\|_{\widetilde{L}_{T}^{q_{2}\left(\dot{B}_{p, 1}^{s_{1}^{\prime}}\right)}}\right),
$$

and if $s_{1}, s_{1}^{\prime} \leq \frac{3}{p}-1$, then

$$
\|f g\|_{\widetilde{L}_{T}^{q}\left(\dot{B}_{2,2}^{s_{1}+s_{2}-\frac{3}{p}}(\omega)\right)} \leq C\left(\|f\|_{\widetilde{L}_{T}^{q_{1}}\left(\dot{B}_{p, 1}^{s_{1}}(\omega)\right)}\|g\|_{\widetilde{L}_{T}^{q_{2}\left(\dot{B}_{2,2}^{s_{2}}\right)}}+\|f\|_{\widetilde{L}_{T}^{q_{1}}\left(\dot{B}_{2,2}^{s_{2}^{\prime}}\right)}\|g\|_{\widetilde{L}_{T}^{q_{2}}\left(\dot{B}_{p, 1}^{s_{1}^{\prime}}(\omega)\right)}\right) .
$$

Lemma 5.9 Let $p \geq 2$ and $s>-\frac{3}{p}$. Then there holds

$$
\begin{aligned}
& \left\|2^{j s}\right\|\left[\Delta_{j}, f\right] \nabla g\left\|_{L_{T}^{1}\left(L^{2}\right)}\right\|_{\ell^{2}} \leq C\left(\|f\|_{\tilde{L}_{T}^{\infty}\left(\dot{B}_{p, 1}^{\frac{3}{p}}(\omega)\right)}\|g\|_{\tilde{L}_{T}^{1}\left(\dot{B}_{2,2}^{s+1}\right)}+\|f\|_{\tilde{L}_{T}^{\infty}\left(\dot{B}_{2,2}^{s}(\omega)\right)}\|g\|_{\tilde{L}_{T}^{1}\left(\dot{B}_{p, 1}^{\frac{3}{p}}\right)}\right), \\
& \left\|2^{j s} \omega_{j}(T)\right\|\left[\Delta_{j}, f\right] \nabla g\left\|_{L_{T}^{1}\left(L^{2}\right)}\right\|_{\ell^{2}} \leq C\left(\|f\|_{\tilde{L}_{T}^{\infty}\left(\dot{B}_{p, 1}^{\frac{3}{p}}(\omega)\right)}\|g\|_{\tilde{L}_{T}^{1}\left(\dot{B}_{2,2}^{s+1}\right)}+\|f\|_{\tilde{L}_{T}^{\infty}\left(\dot{B}_{2,2}^{s}(\omega)\right)}\|g\|_{\tilde{L}_{T}^{1}\left(\dot{B}_{p, 1}^{\frac{3}{p}}\right)}\right) .
\end{aligned}
$$

Lemma 5.10 Let $p \geq 2$ and $s>-\frac{3}{p}$. Then there holds

$$
\left\|2^{j s}\right\|\left[\Delta_{j}, f\right] \nabla g\left\|_{L_{T}^{1}\left(L^{2}\right)}\right\|_{\ell^{2}} \leq C\left(\|f\|_{\tilde{L}_{T}^{1}\left(\dot{B}_{p, 1}^{\frac{3}{p}+1}\right)}\|g\|_{\tilde{L}_{T}^{\infty}\left(\dot{B}_{2,2}^{s}\right)}+\|f\|_{\tilde{L}_{T}^{1}\left(\dot{B}_{2,2}^{s+1}\right)}\|g\|_{\tilde{L}_{T}^{\infty}\left(\dot{B}_{p, 1}^{\frac{3}{p}}\right)}\right) .
$$

\section{Hoff's energy method}

In a series of papers [22, 23, 24, 25], Hoff developed a method to construct global weak solution for the discontinuous initial data with small energy. An important property of this solution is that the density does not develop vacuum and can not concentrate if the initial density is bounded below and above. In this section, we will adapt his method to our case. Due to the stronger condition on the initial data, the restriction on the viscosity coefficient can be removed and the obtained estimate is also better.

We set $\sigma(t)=\min (1, t)$ and define

$$
\begin{aligned}
& A_{1}(T)=\sup _{t \in[0, T]}\|\nabla u(t)\|_{L^{2}}^{2}+\int_{0}^{T} \int_{\mathbf{R}^{3}} \rho|\dot{u}|^{2} d x d t, \\
& A_{2}(T)=\sup _{t \in[0, T]} \sigma(t) \int_{\mathbf{R}^{3}} \rho|\dot{u}(t, x)|^{2} d x+\int_{0}^{T} \int_{\mathbf{R}^{3}} \sigma|\nabla \dot{u}|^{2} d x d t .
\end{aligned}
$$

Here and what follows, we denote

$$
\dot{f}=f_{t}+u \cdot \nabla f .
$$

Theorem 6.1 Let $(\rho, u)$ be a solution of (1.1) satisfying

$$
\rho-\bar{\rho} \in C\left([0, T] ; H^{2}\right), \quad u \in C\left([0, T] ; H^{2}\right) \cap L^{2}\left(0, T ; H^{3}\right) .
$$

There exists a constant $\varepsilon_{0}$ depending only on $\lambda, \mu, c_{0}, \bar{\rho}$ such that if the initial data $\left(\rho_{0}, u_{0}\right)$ satisfies

$$
\begin{aligned}
& c_{0} \leq \rho_{0}(x) \leq c_{0}^{-1}, \quad x \in \mathbf{R}^{3}, \\
& \int_{\mathbf{R}^{3}}\left|\rho_{0}(x)-\bar{\rho}\right|^{2} d x+\left\|u_{0}\right\|_{H^{1}}^{2}=C_{0} \leq \varepsilon_{0},
\end{aligned}
$$


then we have

$$
\begin{aligned}
& \frac{c_{0}}{2} \leq \rho(t, x) \leq 2 c_{0}^{-1}, \quad(t, x) \in[0, T] \times \mathbf{R}^{3}, \\
& A_{1}(T)+A_{2}(T) \leq C_{0}^{\frac{1}{2}} .
\end{aligned}
$$

Proof. Due to the assumption, there exists a $0<T_{0} \leq T$ such that the solution $(\rho, u)$ satisfies

$$
\begin{aligned}
& \frac{c_{0}}{2} \leq \rho(t, x) \leq 2 c_{0}^{-1}, \quad(t, x) \in\left[0, T_{0}\right] \times \mathbf{R}^{3}, \\
& A_{1}\left(T_{0}\right)+A_{2}\left(T_{0}\right) \leq C_{0}^{\frac{1}{2}} .
\end{aligned}
$$

Without loss of generality, we assume that $T_{0}$ is a maximal time so that the above inequalities hold. In the following, we will give a refined estimate on $\left[0, T_{0}\right]$ for the solution. We denote $C$ by a constant depending only on $\lambda, \mu, c_{0}, \bar{\rho}$.

Step 1. $L^{2}$ energy estimate

$$
\begin{aligned}
& \int_{\mathbf{R}^{3}} \frac{1}{2} \rho(t, x)|u(t, x)|^{2}+G(\rho(t, x)) d x+\int_{0}^{t} \int_{\mathbf{R}^{3}}|\nabla u|^{2} d x d t \\
& \leq \int_{\mathbf{R}^{3}} \frac{1}{2} \rho_{0}\left|u_{0}\right|^{2}+G\left(\rho_{0}\right) d x \leq C C_{0},
\end{aligned}
$$

where $G(\rho)$ is the potential energy density defined by

$$
G(\rho)=\rho \int_{\bar{\rho}}^{\rho} \frac{P(s)-P(\bar{\rho})}{s^{2}} d s .
$$

Due to (1.4), we have

$$
|g(\rho)| \leq C G(\rho)
$$

if $g \in C^{2}$ with $g(\bar{\rho})=g^{\prime}(\bar{\rho})=0$.

Step 2. $H^{1}$ energy estimate

Multiply (1.1) by $\dot{u}$ and integrate the resulting equation over $\mathbf{R}^{3}$ to obtain

$$
\int_{\mathbf{R}^{3}} \rho|\dot{u}|^{2} d x=\int_{\mathbf{R}^{3}}(-\dot{u} \cdot \nabla P+\mu \Delta u \cdot \dot{u}+\lambda \nabla \operatorname{div} u \cdot \dot{u}) d x .
$$

By the continuity equation, we have

$$
\partial_{t} P+\operatorname{div}(u P)=\operatorname{div} u\left(P-P^{\prime} \rho\right) .
$$

Then integration by parts yields that

$$
\begin{aligned}
& \int_{\mathbf{R}^{3}}-\dot{u} \cdot \nabla P d x=\int_{\mathbf{R}^{3}}\left(\operatorname{div} u_{t}(P-P(\bar{\rho}))-(u \cdot \nabla u) \cdot \nabla P\right) d x \\
& =\partial_{t} \int_{\mathbf{R}^{3}} \operatorname{div} u(P-P(\bar{\rho})) d x+\int_{\mathbf{R}^{3}}\left(\left(P^{\prime} \rho-P\right)(\operatorname{div} u)^{2}+P \partial_{i} u^{j} \partial_{j} u^{i}\right) d x \\
& \leq \partial_{t} \int \operatorname{div} u\left(P-P(\bar{\rho}) d x+C\|\nabla u\|_{2}^{2} .\right.
\end{aligned}
$$


Integration by parts again gives

$$
\begin{aligned}
\mu \int_{\mathbf{R}^{3}} \Delta u \cdot \dot{u} d x & =-\frac{\mu}{2} \partial_{t} \int_{\mathbf{R}^{3}}|\nabla u|^{2} d x-\mu \int_{\mathbf{R}^{3}} \partial_{i} u^{j} \partial_{i}\left(u^{k} \partial_{k} u^{j}\right) d x \\
& \leq-\frac{\mu}{2} \partial_{t} \int_{\mathbf{R}^{3}}|\nabla u|^{2} d x+C \int|\nabla u|^{3} d x
\end{aligned}
$$

and similarly,

$$
\begin{aligned}
\lambda \int_{\mathbf{R}^{3}} \nabla \operatorname{div} u \cdot \dot{u} d x & =-\frac{\lambda}{2} \partial_{t} \int_{\mathbf{R}^{3}}|\operatorname{div} u|^{2} d x-\lambda \int_{\mathbf{R}^{3}} \partial_{i} u^{j} \partial_{i}\left(u^{k} \partial_{k} u^{j}\right) d x \\
& \leq-\frac{\lambda}{2} \partial_{t} \int_{\mathbf{R}^{3}}|\operatorname{div} u|^{2} d x+C \int_{\mathbf{R}^{3}}|\nabla u|^{3} d x .
\end{aligned}
$$

And by (6.1) and (6.2), we have

$$
\int_{\mathbf{R}^{3}} \operatorname{div} u\left(P-P(\bar{\rho}) d x \leq\|\operatorname{div} u\|_{2}\|P-P(\bar{\rho})\|_{2} \leq C C_{0}^{1 / 2}\|\operatorname{div} u\|_{2} .\right.
$$

Plugging (6.5)-(6.7) into (6.3) yields that

$$
A_{1}\left(T_{0}\right) \leq C C_{0}+C \int_{0}^{T_{0}} \int|\nabla u|^{3} d x d t .
$$

Step 3. $H^{2}$ energy estimate

We take the material derivative to the second equation of (1.1) to obtain

$$
\begin{aligned}
& \rho \dot{u}_{t}+\rho u \cdot \nabla \dot{u}+\nabla P_{t}+\operatorname{div}(\nabla P \otimes u) \\
& \quad=\mu\left[\Delta u_{t}+\operatorname{div}(\Delta u \otimes u)\right]+\lambda\left[\nabla \operatorname{div} u_{t}+\operatorname{div}((\nabla \operatorname{div} u) \otimes u)\right] .
\end{aligned}
$$

Let us introduce some notations. We denote $\operatorname{div}(f \otimes u)=\sum_{j=1}^{3} \partial_{j}\left(f u_{j}\right)$. For two matrices $A=\left(a_{i j}\right)_{3 \times 3}$ and $B=\left(b_{i j}\right)_{3 \times 3}$, we use the notation $A: B=\sum_{i, j=1}^{3} a_{i j} b_{i j}$ and $A B$ is as usual the multiplication of matrix.

Multiply the above equation by $\sigma(t) \dot{u}$ and integrate by parts to get

$$
\begin{aligned}
& \left(\frac{\sigma}{2} \int_{\mathbf{R}^{3}} \rho|\dot{u}|^{2} d x\right)_{t}-\frac{1}{2} \sigma^{\prime} \int_{\mathbf{R}^{3}} \rho|\dot{u}|^{2} d x \\
& =-\int_{\mathbf{R}^{3}} \sigma \dot{u}^{j}\left[\partial_{j} P_{t}+\operatorname{div}\left(\partial_{j} P u\right)\right] d x+\mu \sigma \int_{\mathbf{R}^{3}} \dot{u} \cdot\left(\Delta u_{t}+\operatorname{div}(\Delta u \otimes u)\right) d x \\
& \quad+\lambda \sigma \int_{\mathbf{R}^{3}} \dot{u} \cdot\left[\nabla \operatorname{div} u_{t}+\operatorname{div}((\nabla \operatorname{div} u) \otimes u)\right] d x .
\end{aligned}
$$

Now integration by parts and use (6.4) to obtain

$$
\begin{aligned}
& -\int_{\mathbf{R}^{3}} \sigma \dot{u}^{j}\left[\partial_{j} P_{t}+\operatorname{div}\left(\partial_{j} P u\right)\right] d x=-\int_{\mathbf{R}^{3}} \sigma\left[\left(u \cdot \nabla P+P^{\prime} \rho \operatorname{div} u\right) \partial_{j} \dot{u}^{j}-\partial_{k} \dot{u}^{j} \partial_{j} P u^{k}\right] d x \\
& =-\int_{\mathbf{R}^{3}} \sigma\left[P^{\prime} \rho \operatorname{div} u \partial_{j} \dot{u}^{j}-P \partial_{k}\left(u^{k} \partial_{j} \dot{u}^{j}\right)+P \partial_{j}\left(\partial_{k} \dot{u}^{j} u^{k}\right)\right] d x \\
& \leq C \sigma\|\nabla u\|_{2}\|\nabla \dot{u}\|_{2} .
\end{aligned}
$$


Integrate by parts again to obtain

$$
\begin{aligned}
& \mu \int_{\mathbf{R}^{3}} \dot{u} \cdot\left(\Delta u_{t}+\operatorname{div}(\Delta u \otimes u)\right) d x=-\mu \int_{\mathbf{R}^{3}}\left[\nabla \dot{u}: \nabla u_{t}+u \otimes \Delta u: \nabla \dot{u}\right] d x \\
& =-\mu \int_{\mathbf{R}^{3}}\left[|\nabla \dot{u}|^{2}-\nabla(u \cdot \nabla u): \nabla \dot{u}+u \otimes \Delta u: \nabla \dot{u}\right] d x \\
& =-\mu \int_{\mathbf{R}^{3}}\left[|\nabla \dot{u}|^{2}-((\nabla u \nabla u)+(u \cdot \nabla) \nabla u): \nabla \dot{u}-\nabla(u \cdot \nabla \dot{u}): \nabla u\right] d x \\
& =-\mu \int_{\mathbf{R}^{3}}\left[|\nabla \dot{u}|^{2}-(\nabla u \nabla u): \nabla \dot{u}-\operatorname{div}(\nabla u \otimes u): \nabla \dot{u}-(\nabla u \nabla \dot{u}): \nabla u-((u \cdot \nabla) \nabla \dot{u}): \nabla u\right] d x \\
& =-\mu \int_{\mathbf{R}^{3}}\left[|\nabla \dot{u}|^{2}-(\nabla u \nabla u): \nabla \dot{u}+((u \cdot \nabla) \nabla \dot{u}): \nabla u-(\nabla u \nabla \dot{u}): \nabla u-((u \cdot \nabla) \nabla \dot{u}): \nabla u\right] d x \\
& \leq \int_{\mathbf{R}^{3}}\left[-\frac{3 \mu}{4}|\nabla \dot{u}|^{2}+C|\nabla u|^{4}\right] d x .
\end{aligned}
$$

To estimate the last term, note that

$$
\begin{aligned}
& \operatorname{div}((\nabla \operatorname{div} u) \otimes u)=\nabla(u \cdot \nabla \operatorname{div} u)-\operatorname{div}(\operatorname{div} u \nabla \otimes u)+\nabla(\operatorname{div} u)^{2}, \\
& \operatorname{div} \dot{u}=\operatorname{div} u_{t}+\operatorname{div}(u \cdot \nabla u)=\operatorname{div} u_{t}+u \cdot \nabla \operatorname{div} u+\nabla u:(\nabla u)^{\prime} .
\end{aligned}
$$

Here $A^{\prime}$ means the transpose of matrix $A$. We have

$$
\begin{aligned}
& \lambda \int_{\mathbf{R}^{3}} \dot{u} \cdot\left[\nabla \operatorname{div} u_{t}+\operatorname{div}((\nabla \operatorname{div} u) \otimes u)\right] d x \\
& =-\lambda \int_{\mathbf{R}^{3}}\left[\operatorname{div} \dot{u} \operatorname{div} u_{t}+\operatorname{div} \dot{u}(u \cdot \nabla \operatorname{div} u)-\operatorname{div} u(\nabla \dot{u})^{\prime}: \nabla u+\operatorname{div} \dot{u}(\operatorname{div} u)^{2}\right] d x \\
& =-\lambda \int_{\mathbf{R}^{3}}\left[|\operatorname{div} \dot{u}|^{2}-\operatorname{div} \dot{u} \nabla u:(\nabla u)^{\prime}-\operatorname{div} u(\nabla \dot{u})^{\prime}: \nabla u+\operatorname{div} \dot{u}(\operatorname{div} u)^{2}\right] d x \\
& \leq \int_{\mathbf{R}^{3}}\left[-\frac{\lambda}{2}|\operatorname{div} \dot{u}|^{2}+\frac{1}{4}|\nabla \dot{u}|^{2}+C|\nabla u|^{4}\right] d x .
\end{aligned}
$$

Summing up the above estimates, we obtain

$$
A_{2}\left(T_{0}\right) \leq C C_{0}+C A_{1}\left(T_{0}\right)+C \int_{0}^{T_{0}} \int_{\mathbf{R}^{3}} \sigma|\nabla u|^{4} d x d t,
$$

which along with (6.8) gives

$$
A_{1}\left(T_{0}\right)+A_{2}\left(T_{0}\right) \leq C C_{0}+C \int_{0}^{T_{0}} \int_{\mathbf{R}^{3}} \sigma|\nabla u|^{4} d x d t+C \int_{0}^{T_{0}} \int_{\mathbf{R}^{3}}|\nabla u|^{3} d x d t .
$$

It remains to bound the right hand side. For this purpose, we set

$$
F=(\mu+\lambda) \operatorname{div} u-P(\rho)+P(\bar{\rho}), \quad \omega=\nabla \times u .
$$

By (6.4), we have

$$
\begin{aligned}
& \partial_{t}(P-P(\bar{\rho}))+u \cdot \nabla(P-P(\bar{\rho})) \\
& =\frac{P^{\prime} \rho}{P}\left[\frac{1}{\mu+\lambda} F(P(\rho)-P(\bar{\rho}))+\frac{1}{\mu+\lambda}(P-P(\bar{\rho}))^{2}+P(\bar{\rho}) \operatorname{div} u\right],
\end{aligned}
$$


which implies that

$$
\begin{aligned}
& C\|P(\rho)-P(\bar{\rho})\|_{4}^{4} \\
& \leq \partial_{t} \int(P-P(\bar{\rho}))^{3} d x+\delta\|P(\rho)-P(\bar{\rho})\|_{4}^{4}+C\left(\|F\|_{4}^{4}+\|\nabla u\|_{2}^{2}\right) .
\end{aligned}
$$

Taking $\delta$ small and integrating the above equation on $\left[0, T_{0}\right]$, we get

$$
\begin{aligned}
\int_{0}^{T_{0}} \sigma\|P(\rho)-P(\bar{\rho})\|_{L^{4}}^{4} d t \leq & C \sup _{t \in[0, T]}\|P(\rho)-P(\bar{\rho})\|_{L^{3}}^{3}+C \int_{0}^{\sigma(T)}\|P(\rho)-P(\bar{\rho})\|_{L^{3}}^{3} d t \\
& +C \int_{0}^{T_{0}} \sigma\|F\|_{4}^{4} d t+C C_{0} \\
\leq & C C_{0}+C \int_{0}^{T_{0}} \sigma\|F\|_{L^{4}}^{4} d t
\end{aligned}
$$

from which and Lemma 6.2, we infer that

$$
\begin{aligned}
& \int_{0}^{T_{0}} \int_{\mathbf{R}^{3}} \sigma|\nabla u|^{4} d x d t \\
& \leq C \int_{0}^{T_{0}} \sigma\left(\|F\|_{4}^{4}+\|\omega\|_{4}^{4}\right) d t+C \int_{0}^{T_{0}} \sigma\|P(\rho)-P(\bar{\rho})\|_{4}^{4} d t \\
& \leq C \int_{0}^{T_{0}} \sigma\left(\|\nabla u\|_{2}+\|P(\rho)-P(\bar{\rho})\|_{2}\right)\|\rho \dot{u}\|_{2}^{3} d t+C C_{0} \\
& \leq C \sup _{t \in\left[0, T_{0}\right]}\left(\sigma^{1 / 2}\left\|\rho^{1 / 2} \dot{u}\right\|_{2}\left(\|\nabla u\|_{2}+C_{0}^{1 / 2}\right)\right) \int_{0}^{T_{0}}\left\|\rho^{1 / 2} \dot{u}\right\|_{2}^{2} d t+C C_{0} \\
& \leq C\left(A_{1}^{1 / 2}+C_{0}^{1 / 2}\right) A_{2}^{1 / 2} A_{1}(T)+C_{0} \leq C C_{0},
\end{aligned}
$$

and

$$
\begin{aligned}
& \int_{0}^{T_{0}} \int_{\mathbf{R}^{3}}|\nabla u|^{3} d x d t \\
& \leq \int_{0}^{\sigma\left(T_{0}\right)} \int_{\mathbf{R}^{3}}|\nabla u|^{3} d x d t+\int_{\sigma\left(T_{0}\right)}^{T_{0}} \int_{\mathbf{R}^{3}}|\nabla u|^{3} d x d t \\
& \leq \int_{0}^{\sigma\left(T_{0}\right)}\|\nabla u\|_{2}^{\frac{3}{2}}\left(\|\rho \dot{u}\|_{2}^{\frac{3}{2}}+\|P-P(\bar{\rho})\|_{2}^{\frac{3}{2}}\right) d x d t+\int_{\sigma\left(T_{0}\right)}^{T_{0}} \int_{\mathbf{R}^{3}}\left(|\nabla u|^{4}+|\nabla u|^{2}\right) d x d t \\
& \leq C \sup _{t \in\left[0, \sigma\left(T_{0}\right)\right]}\|\nabla u\|_{2}^{\frac{3}{2}} \int_{0}^{\sigma\left(T_{0}\right)}\|\rho \dot{u}\|_{2}^{\frac{3}{2}}+C C_{0} \\
& \leq C A_{1}^{\frac{3}{2}}+C C_{0} \leq C C_{0}^{\frac{3}{4}} .
\end{aligned}
$$

Plugging them into (6.10) and taking $\varepsilon$ small enough depending on $\mu, \lambda, \bar{\rho}, c_{0}$, we conclude

$$
A_{1}\left(T_{0}\right)+A_{2}\left(T_{0}\right) \leq \frac{1}{2} C_{0}^{\frac{1}{2}}
$$

Step 4. Lower and upper bound of the density 
Set $L=\log (\rho)$, which satisfies

$$
(2 \mu+\lambda) \dot{L}+(P(\rho)-P(\bar{\rho}))=-F .
$$

For $0<t<\sigma\left(T_{0}\right)$, we have

$$
\begin{aligned}
\int_{0}^{\sigma\left(T_{0}\right)}\|F\|_{L^{\infty}} d t & \leq C \int_{0}^{\sigma\left(T_{0}\right)}\|F\|_{L^{6}}^{\frac{1}{2}}\|\nabla F\|_{L^{6}}^{\frac{1}{2}} d t \\
& \leq C \int_{0}^{\sigma\left(T_{0}\right)}\|\rho \dot{u}\|_{L^{2}}^{\frac{1}{2}}\|\rho \dot{u}\|_{L^{6}}^{\frac{1}{2}} d t \\
& \leq C \int_{0}^{\sigma\left(T_{0}\right)}\left\|\rho^{1 / 2} \dot{u}\right\|_{L^{2}}^{\frac{1}{2}}\|\nabla \dot{u}\|_{L^{2}}^{\frac{1}{2}} d t \\
& =C \int_{0}^{\sigma\left(T_{0}\right)}\left(t^{-1 / 2}\right)^{\frac{1}{2}}\left(\left\|\rho^{1 / 2} \dot{u}\right\|_{L^{2}}^{2}\right)^{\frac{1}{4}}\left(t\|\nabla \dot{u}\|_{L^{2}}^{2}\right)^{\frac{1}{4}} d t \\
& \leq C C_{0}^{\frac{1}{4}},
\end{aligned}
$$

which implies that for $t \leq \sigma\left(T_{0}\right)$,

$$
\inf \left(\log \rho_{0}(x)\right)-C C_{0}^{\frac{1}{4}}-C t \leq \log \rho(t, x) \leq \sup \left(\log \rho_{0}(x)\right)+C C_{0}^{\frac{1}{4}}+C t,
$$

hence, one can choose $\varepsilon_{0}, \tau$ sufficiently small such that for $t \leq \tau \leq \sigma\left(T_{0}\right)$,

$$
\frac{3}{4} c_{0}<\rho(t, x)<\frac{3}{2} c_{0}^{-1} .
$$

For $\tau \leq t_{1} \leq t_{2} \leq T_{0}$, we have

$$
\begin{aligned}
\int_{t_{1}}^{t_{2}}\|F\|_{L^{\infty}} d t & \leq C \int_{t_{1}}^{t_{2}}\left\|\rho^{1 / 2} \dot{u}\right\|_{L^{2}}^{\frac{1}{2}}\|\nabla \dot{u}\|_{L^{2}}^{\frac{1}{2}} d t \\
& \leq C\left(t_{2}-t_{1}\right)^{1 / 2} \int_{t_{1}}^{t_{2}}\left(\left\|\rho^{1 / 2} \dot{u}\right\|_{L^{2}}^{2}+\|\nabla \dot{u}\|_{L^{2}}^{2}\right) d t \\
& \leq \eta\left(t_{2}-t_{1}\right)+C C_{0}
\end{aligned}
$$

for any $\eta>0$. Now let $T^{*}$ be a maximal time so that (6.12) holds. If $T^{*} \geq T$, then we are done. Otherwise, we have

$$
\rho\left(T^{*}, x\left(T^{*}\right)\right)=\frac{3}{2} c_{0}^{-1} \quad \text { or } \quad \rho\left(T^{*}, x\left(T^{*}\right)\right)=\frac{3}{4} c_{0},
$$

where the curve $x(t)$ is defined by

$$
\frac{d}{d t} x(t)=u(t, x(t)), \quad x(0)=x .
$$

If $\rho\left(T^{*}, x\left(T^{*}\right)\right)=\frac{3}{2} c_{0}^{-1}$, then there exits $T^{*}>T_{1} \geq \tau$ such that

$$
\rho\left(T_{1}, x\left(T_{1}\right)\right)=c_{0}^{-1}, \quad c_{0}^{-1} \leq \rho(t, x(t)) \leq \frac{3}{2} c_{0}^{-1} \quad \text { for } \quad t \in\left[T_{1}, T^{*}\right] .
$$


Then we conclude by (1.4) that

$$
L\left(T^{*}, x\left(T^{*}\right)\right)-L\left(T_{1}, x\left(T_{1}\right)\right) \leq C C_{0},
$$

if we choose $\eta$ small enough. If we choose $\varepsilon_{0}$ sufficiently small, this in turn implies that

$$
\rho\left(T^{*}, x\left(T^{*}\right)\right)<\frac{3}{2} c_{0}^{-1},
$$

which contradicts with the definition of $T^{*}$. Similar argument yields that

$$
\rho\left(T^{*}, x\left(T^{*}\right)\right)>\frac{3}{4} c_{0}
$$

if $\rho\left(T^{*}, x\left(T^{*}\right)\right)=\frac{3}{4} c_{0}$. Hence, we have

$$
\frac{3}{4} c_{0}<\rho(t, x)<\frac{3}{2} c_{0}^{-1}, \quad \text { for } \quad 0 \leq t \leq T_{0} .
$$

With (6.11) and (6.13), we conclude the proof by continuity argument.

Lemma 6.2 For any $p \in[2,6]$, there exists a constant $C$ depending only on $\mu, \lambda$ such that

$$
\begin{aligned}
& \|\nabla u\|_{p} \leq C\left(\|F\|_{p}+\|\omega\|_{p}+\|P(\rho)-P(\bar{\rho})\|_{p}\right), \\
& \|\nabla u\|_{p} \leq C\|\nabla u\|_{2}^{\frac{6-p}{2 p}}\left(\|\rho \dot{u}\|_{2}+\|P(\rho)-P(\bar{\rho})\|_{6}\right)^{\frac{3 p-6}{2 p}} .
\end{aligned}
$$

Proof. By the elliptic estimate and the definition of $F$, we have

$$
\|\nabla u\|_{p} \leq C\left(\|\operatorname{div} u\|_{p}+\|\omega\|_{p}\right) \leq C\left(\|F\|_{p}+\|\omega\|_{p}+\|P(\rho)-P(\bar{\rho})\|_{p}\right) .
$$

Noting that

$$
\Delta F=\operatorname{div}(\rho \dot{u}), \quad \mu \Delta \omega=\nabla \times(\rho \dot{u}),
$$

we have by the elliptic estimate that

$$
\|\nabla F\|_{2}+\|\nabla \omega\|_{2} \leq C\|\rho \dot{u}\|_{2}
$$

Then by Hölder inequality and Sobolev inequality, we get

$$
\begin{aligned}
\|\nabla u\|_{p} & \leq C\|\nabla u\|_{2}^{\frac{6-p}{2 p}}\|\nabla u\|_{6}^{\frac{3 p-6}{2 p}} \\
& \leq C\|\nabla u\|_{2}^{\frac{6-p}{2 p}}\left(\|F\|_{6}+\|\omega\|_{6}+\|P(\rho)-P(\bar{\rho})\|_{6}\right)^{\frac{3 p-6}{2 p}} \\
& \leq C\|\nabla u\|_{2}^{\frac{6-p}{2 p}}\left(\|\rho \dot{u}\|_{2}+\|P(\rho)-P(\bar{\rho})\|_{6}\right)^{\frac{3 p-6}{2 p}}
\end{aligned}
$$

This finishes the proof of lemma. 


\section{Continuation criterion}

In order to extend a local solution to a global one, we need to establish a continuation criterion of smooth solution. The proof is motivated by [33].

Theorem 7.1 Let $(\rho, u)$ be a solution of (1.1) satisfying

$$
\rho(0)>0, \quad \rho-\bar{\rho} \in C\left([0, T] ; H^{2}\right), \quad u \in C\left([0, T] ; H^{2}\right) \cap L^{2}\left(0, T ; H^{3}\right) .
$$

Let $T^{*}$ be the maximal existence time of the solution. If $T^{*}<+\infty$, then it is necessary

$$
\limsup _{t \uparrow T^{*}}\left(\|\rho(t)\|_{\infty}+\|u(t)\|_{q}\right)=+\infty
$$

for any $q>3$.

Remark 7.2 Compared with Theorem 1.3 in [33], the restriction on the viscosity coefficient $(\lambda, \mu)$ is removed, but the price to pay is to impose the condition on the velocity $u$. Due to $\rho(0)>0$, the compatibility condition on the initial data is also removed.

Proof. We use the contradiction argument. Hence, assume that $T^{*}<\infty$ and

$$
\sup _{t \in\left[0, T^{*}\right)}\left(\|\rho(t)\|_{\infty}+\|u(t)\|_{q}\right)=M<+\infty
$$

In what follows, we denote $C$ by a constant depending on $T, M,\left\|u_{0}\right\|_{H^{2}}$ and $\left\|\rho_{0}-\bar{\rho}\right\|_{H^{2}}$.

First of all, we have

$$
\int_{\mathbf{R}^{3}} \frac{1}{2} \rho(t, x)|u(t, x)|^{2}+G(\rho(t, x)) d x+\int_{0}^{t} \int_{\mathbf{R}^{3}}|\nabla u|^{2} d x d t \leq C .
$$

This gives by (7.2) and (6.2) that for any $r \in[2, \infty]$,

$$
\|\rho-\bar{\rho}\|_{L^{\infty}\left(0, T ; L^{r}\right)}+\|\sqrt{\rho} u\|_{L^{\infty}\left(0, T ; L^{2}\right)}+\|\nabla u\|_{L^{2}\left(0, T ; L^{2}\right)} \leq C .
$$

Let $v=L^{-1} \nabla P(\rho)$ be a solution of the following elliptic system

$$
L v \stackrel{\text { def }}{=} \mu \Delta v+\lambda \nabla \operatorname{div} v=\nabla P(\rho) .
$$

By elliptic estimate and (7.3) , for $r \in[2, \infty)$

$$
\|\nabla v\|_{r} \leq C\|P(\rho)-P(\bar{\rho})\|_{r} \leq C, \quad\left\|\nabla^{2} v\right\|_{r} \leq C\|\nabla \rho\|_{r}
$$

Introduce a new unknown $w=u-v$. Note that $\operatorname{div} w=(\lambda+\mu) \operatorname{div} u-P(\rho)+P(\bar{\rho})$ is a so called the effective viscous flux introduced by Hoff [22]. It is easy to find that $w$ satisfies

$$
\rho \partial_{t} w-\mu \Delta w-\lambda \nabla \operatorname{div} w=\rho F
$$

with

$$
F=-u \cdot \nabla u+L^{-1} \nabla \operatorname{div}(p(\rho) u)+L^{-1} \nabla\left(\left(\rho p^{\prime}(\rho)-p(\rho)\right) \operatorname{div} u\right) .
$$


Multiplying the above equation by $\partial_{t} w$ and integrating by parts, we obtain

$$
\frac{d}{d t} \int_{\mathbf{R}^{3}} \mu|\nabla w|^{2}+\lambda|\operatorname{div} w|^{2} d x+\frac{1}{2} \int_{\mathbf{R}^{3}} \rho\left|\partial_{t} w\right|^{2} d x \leq \frac{1}{2}\|\sqrt{\rho} F\|_{2}^{2} .
$$

By Sobolev inequality, interpolation inequality, (7.2) and (7.4), we get

$$
\begin{aligned}
\|\sqrt{\rho} u \cdot \nabla u\|_{L^{2}(\Omega)} & \leq C\|u\|_{q}\|\nabla u\|_{\frac{2 q}{q-2}} \leq C\left(\|\nabla w\|_{\frac{2 q}{q-2}}+\|\nabla v\|_{\frac{2 q}{q-2}}\right) \\
& \leq C\|\nabla w\|_{2}+\epsilon\left\|\nabla^{2} w\right\|_{2}+C \\
& \leq C+C\|\nabla u\|_{2}+\epsilon\left\|\nabla^{2} w\right\|_{2}
\end{aligned}
$$

for any $\epsilon>0$, and by elliptic estimate, (7.3) and (7.4),

$$
\left\|\sqrt{\rho} L^{-1} \nabla \operatorname{div}[p(\rho) u]\right\|_{2} \leq C\|p(\rho) u\|_{2} \leq C\|\sqrt{\rho} u\|_{2} \leq C,
$$

and by Sobolev inequality,

$$
\begin{aligned}
& \left\|\sqrt{\rho} L^{-1} \nabla\left(\rho p^{\prime}-p\right) \operatorname{div} u\right\|_{2} \\
& \leq\|\rho-\bar{\rho}\|_{3}\left\|L^{-1} \nabla\left(\rho p^{\prime}-p\right) \operatorname{div} u\right\|_{6}+C\|(\rho-\bar{\rho}) \operatorname{div} u\|_{\frac{6}{5}}+C \bar{\rho}\|u\|_{2} \\
& \leq C\|\nabla u\|_{2}+C\|\sqrt{\rho} u\|_{2} \leq C+C\|\nabla u\|_{2},
\end{aligned}
$$

here we used

$$
\bar{\rho}^{2} \int_{\mathbf{R}^{3}}|u|^{2} d x \leq 2 \int_{\mathbf{R}^{3}}|\rho-\bar{\rho}|^{2}|u|^{2} d x+2 \int_{\mathbf{R}^{3}} \rho^{2}|u|^{2} d x .
$$

Hence,

$$
\|\sqrt{\rho} F\|_{2} \leq C\left(1+\|\nabla u\|_{2}\right)+\epsilon\left\|\nabla^{2} w\right\|_{2} .
$$

Noting that $L w=\rho \partial_{t} w-\rho F$, we get by elliptic estimate that

$$
\left\|\nabla^{2} w\right\|_{2} \leq C\left(\left\|\rho \partial_{t} w\right\|_{2}+\|\rho F\|_{2}\right) \leq C\left(\left\|\sqrt{\rho} \partial_{t} w\right\|_{2}+\|\sqrt{\rho} F\|_{2}\right),
$$

which implies by taking $\epsilon$ small enough that

$$
\|\sqrt{\rho} F\|_{2} \leq C\left(1+\|\nabla u\|_{2}\right)+\frac{1}{2}\left\|\sqrt{\rho} \partial_{t} w\right\|_{2} .
$$

This along with (7.5) gives

$$
\|\nabla w\|_{L^{\infty}\left(0, T ; L^{2}\right)}+\left\|\sqrt{\rho} \partial_{t} w\right\|_{L^{2}\left(0, T ; L^{2}\right)}+\left\|\nabla^{2} w\right\|_{L^{2}\left(0, T ; L^{2}\right)} \leq C,
$$

which in turn implies by (7.4) and Sobolev inequality that

$$
\|\nabla u\|_{L^{\infty}\left(0, T ; L^{2}\right)}+\|\nabla u\|_{L^{2}\left(0, T ; L^{r}\right)} \leq C \quad \text { for } \quad r \in[2,6] .
$$

Now let us turn to the high order energy estimate. Thanks to the proof of Step 3 in Theorem 6.1, we have

$$
\begin{aligned}
& \frac{d}{d t} \int_{\mathbf{R}^{3}} t \rho|\dot{u}|^{2} d x+\mu t \int_{\mathbf{R}^{3}}|\nabla \dot{u}|^{2} d x+\lambda t \int_{\mathbf{R}^{3}}|\operatorname{div} \dot{u}|^{2} d x \\
& \leq C+C t \int_{\mathbf{R}^{3}}|\nabla u|^{4} d x+C \int_{\mathbf{R}^{3}}|\nabla u|^{3} d x .
\end{aligned}
$$


Noting that

$$
\mu \Delta w+\lambda \nabla \operatorname{div} w=\rho \dot{u}
$$

elliptic estimate yields that

$$
\left\|\nabla^{2} w\right\|_{2} \leq C\|\rho \dot{u}\|_{2} \leq C\|\sqrt{\rho} \dot{u}\|_{2} .
$$

Then by Sobolev inequality, (7.4) and (7.6), we infer that

$$
\begin{aligned}
\|\nabla u\|_{4}^{4} & \leq\|\nabla u\|_{2}\|\nabla u\|_{6}^{3} \leq C\|\nabla u\|_{6}^{2}\left(\|\nabla w\|_{6}+\|\nabla v\|_{6}\right) \\
& \leq C\|\nabla u\|_{6}^{2}\left(1+\left\|\nabla^{2} w\right\|_{2}\right) \leq C\|\nabla u\|_{6}^{2}\left(1+\|\sqrt{\rho} \dot{u}\|_{2}\right), \\
\|\nabla u\|_{3}^{3} & \leq\|\nabla u\|_{2}^{\frac{3}{2}}\|\nabla u\|_{6}^{\frac{3}{2}} \leq C\|\nabla u\|_{6}^{\frac{3}{2}} .
\end{aligned}
$$

Plugging them into (17.7) and noting $\|\nabla u(t)\|_{6} \in L^{2}(0, T)$ by (7.6), we deduce by Gronwall's inequality that

$$
t \int_{\mathbf{R}^{3}} \rho|\dot{u}|^{2} d x+\int_{0}^{T} \int_{\mathbf{R}^{3}} t|\nabla \dot{u}|^{2} d x d t \leq C
$$

from which, elliptic estimate and Sobolev inequality, it follows that

$$
\left\|\nabla^{2} w\right\|_{L^{2}\left(0, T ; L^{r}\right)} \leq C\|\rho \dot{u}\|_{L^{2}\left(0, T ; L^{r}\right)} \leq C \quad \text { for } \quad r \in[2,6] .
$$

To obtain the high order estimate for $u$, we need to use the continuity equation. Take the derivative with respect to $x$ for the first equation of (1.1) to obtain

$$
\partial_{t} \nabla \rho+(u \cdot \nabla) \nabla \rho+\nabla u \nabla \rho+\operatorname{div} u \nabla \rho+\rho \nabla \operatorname{div} u=0 .
$$

For $r \in(3,6]$, making $L^{r}$ energy estimate yields that

$$
\begin{aligned}
& \frac{d}{d t} \int_{\mathbf{R}^{3}}|\nabla \rho|^{r} d x \\
& \leq C \int_{\mathbf{R}^{3}}\left|\nabla u \left\|\left.\nabla \rho\right|^{r} d x+C \int_{\mathbf{R}^{3}} \rho|\nabla \operatorname{div} u \| \nabla \rho|^{r-1} d x\right.\right. \\
& \leq C\|\nabla u\|_{\infty}\|\nabla \rho\|_{r}^{r}+C\left\|\nabla^{2} u\right\|_{r}\|\nabla \rho\|_{r}^{r-1} \\
& \leq C\left(\|\nabla w\|_{\infty}+\|\nabla v\|_{\infty}\right)\|\nabla \rho\|_{r}^{r}+C\left(\left\|\nabla^{2} w\right\|_{r}+\left\|\nabla^{2} v\right\|_{r}\right)\|\nabla \rho\|_{r}^{r-1} .
\end{aligned}
$$

Then by (7.4) and Sobolev inequality, we get

$$
\frac{d}{d t} \int_{\mathbf{R}^{3}}|\nabla \rho|^{r} d x \leq C\left(1+\|\nabla v\|_{\infty}+\left\|\nabla^{2} w\right\|_{r}\right)\|\nabla \rho\|_{r}^{r}
$$

and by Lemma 7.3 and elliptic estimate,

$$
\begin{aligned}
\|\nabla v\|_{\infty} & \leq C\left(\|\nabla v\|_{2}+\|\nabla v\|_{\dot{B}_{\infty, \infty}^{0}} \ln \left(e+\|\nabla v\|_{W^{1, r}}\right)\right) \\
& \leq C\left(\|\rho-\bar{\rho}\|_{2}+\|\rho\|_{\infty} \ln \left(e+\|\nabla \rho\|_{r}\right)\right)
\end{aligned}
$$


from which and (7.9), we deduce by Gronwall's inequality that

$$
\|\nabla \rho\|_{r} \leq C
$$

which along with (17.9) and (7.4) gives

$$
\left\|\nabla^{2} u\right\|_{L^{2}\left(0, T ; L^{r}\right)} \leq\left\|\nabla^{2} w\right\|_{L^{2}\left(0, T ; L^{r}\right)}+\left\|\nabla^{2} v\right\|_{L^{2}\left(0, T ; L^{r}\right)} \leq C .
$$

This in turn implies (7.10) also holds for $r \in[2,6]$.

Now we are in position to give $H^{2}$ estimate of $(\rho, u)$. First of all, we have

$$
\frac{d}{d t}\|\rho-\bar{\rho}\|_{H^{2}}^{2} \leq C\left(1+\|\nabla u\|_{\infty}\right)\|\rho-\bar{\rho}\|_{H^{2}}^{2}+C\left\|\nabla^{3} u\right\|_{2}^{2}
$$

While by (17.10),

$$
\|\nabla(\rho \dot{u})\|_{2} \leq\|\nabla \rho\|_{3}\|\dot{u}\|_{6}+C\|\nabla \dot{u}\|_{2} \leq C\|\nabla \dot{u}\|_{2},
$$

this along with elliptic estimate and (7.8) implies that

$$
\left\|\nabla^{2} w(t)\right\|_{2}^{2}+\int_{0}^{t}\left\|\nabla^{3} w(\tau)\right\|_{2}^{2} d \tau \leq C
$$

Indeed, we have

$$
\begin{aligned}
\int_{0}^{t}\left\|\nabla^{3} w(\tau)\right\|_{2}^{2} d \tau & \leq \int_{0}^{\delta}\left\|\nabla^{3} w(\tau)\right\|_{2}^{2} d \tau+\int_{\delta}^{t}\left\|\nabla^{3} w(\tau)\right\|_{2}^{2} d \tau \\
& \leq C+\delta^{-1} \int_{0}^{t} \tau\left\|\nabla^{3} w(\tau)\right\|_{2}^{2} d \tau \leq C
\end{aligned}
$$

Hence by (17.4), we get

$$
\left\|\nabla^{2} u(t)\right\|_{2}^{2}+\int_{0}^{t}\left\|\nabla^{3} u(\tau)\right\|_{2}^{2} d \tau \leq C+C \int_{0}^{t}\left\|\nabla^{2} \rho(\tau)\right\|_{2}^{2} d \tau .
$$

Summing up (7.12) and (7.13), we conclude by Gronwall's inequality that for $0 \leq t<T^{*}$,

$$
\|u(t)\|_{H^{2}}+\|\rho(t)-\bar{\rho}\|_{H^{2}} \leq C .
$$

This ensures that the solution can be continued after $t=T^{*}$.

Lemma 7.3 Let $r>3$ and $u \in W^{1, r}$. Then there holds

$$
\|u\|_{\infty} \leq C\|u\|_{2}+C\|u\|_{\dot{B}_{\infty, \infty}^{0}} \ln \left(e+\|u\|_{W^{1, r}}\right) .
$$

Proof. The proof is standard. For the reader's convenience, we present a proof. We decompose $u$ into

$$
u=S_{-N} u+\sum_{j=-N}^{N} \Delta_{j} u+\sum_{j \geq N+1} \Delta_{j} u
$$


from which and Lemma 2.1, we infer that

$$
\begin{aligned}
\|u\|_{\infty} & \leq 2^{-\frac{3}{2} N}\|u\|_{2}+(2 N+1)\|u\|_{\dot{B}_{\infty, \infty}^{0}}+C \sum_{j \geq N} 2^{\frac{3}{r} j}\left\|\Delta_{j} u\right\|_{r} \\
& \leq 2^{-\frac{3}{2} N}\|u\|_{2}+(2 N+1)\|u\|_{\dot{B}_{\infty, \infty}^{0}}+C 2^{-\left(1-\frac{3}{r}\right) N}\|u\|_{W^{1, r}} .
\end{aligned}
$$

Taking $N \in \mathbf{N}$ such that

$$
2^{-\left(1-\frac{3}{r}\right) N}\|u\|_{W^{1, r}} \sim 1
$$

the desired estimate follows easily.

\section{Proof of Theorem 1.1}

Theorem 4.1, Proposition 5.1 and Proposition 5.2 ensure that there exists a solution $(\rho, u)$ of (1.1) satisfying

$$
\begin{aligned}
& \frac{c_{0}}{2} \leq \rho \leq 2 c_{0}^{-1}, \quad \rho-\bar{\rho} \in \widetilde{L}^{\infty}\left(0, T ; \dot{B}_{p, 1}^{\frac{3}{p}} \cap \dot{B}_{2,2}^{s}\right), \\
& u \in \widetilde{L}^{\infty}\left(0, T ; \dot{B}_{p, 1}^{\frac{3}{p}-1} \cap \dot{B}_{2,2}^{s-1}\right) \cap \widetilde{L}^{1}\left(0, T ; \dot{B}_{p, 1}^{\frac{3}{p}+1} \cap \dot{B}_{2,2}^{s+1}\right) .
\end{aligned}
$$

Moreover, there hold

$$
\begin{aligned}
& \|\rho-\bar{\rho}\|_{\widetilde{L}_{T}^{\infty}\left(\dot{B}_{2,2}^{1-\delta}\right)} \leq C\left(\left\|\rho_{0}-\bar{\rho}\right\|_{\dot{H}^{1-\delta}}+\left\|u_{0}\right\|_{\dot{H}^{-\delta}}\right), \\
& \|u\|_{\widetilde{L}_{T}^{\infty}\left(\dot{B}_{2,2}^{-\delta}\right)} \leq C\left(1+E_{0}\right)\left(\left\|u_{0}\right\|_{\dot{H}^{-\delta}}+T\left\|\rho_{0}-\bar{\rho}\right\|_{\dot{H}^{1-\delta}}\right), \\
& \|u\|_{\widetilde{L}_{T}^{1}\left(\dot{B}_{2,2}^{2-\delta}\right)}+\|u\|_{\widetilde{L}_{T}^{2}\left(\dot{B}_{2,2}^{1-\delta}\right)} \leq C\left(\left\|u_{0}\right\|_{\dot{H}^{-\delta}}+T\left\|\rho_{0}-\bar{\rho}\right\|_{\dot{H}^{1-\delta}}\right), \\
& \|u\|_{\widetilde{L}_{T}^{1}\left(\dot{B}_{p, 1}^{\frac{3}{p}+1}\right)}+\|u\|_{\widetilde{L}_{T}^{2}\left(\dot{B}_{p, 1}^{\frac{3}{p}}\right)} \leq \eta_{2} \leq 1 .
\end{aligned}
$$

and by Remark 5.3 , the existence time $T$ has a lower bound

$$
T \geq \frac{1}{C\left(1+\left\|\rho_{0}-\bar{\rho}\right\|_{\dot{B}_{p, 1}^{\frac{3}{p} \cap H^{s}}}\right)^{\left(s+\frac{1}{2}\right) /\left(s-\frac{3}{2}\right)}}=T_{1} .
$$

Here and what follows, the constant $C$ depends only on $\lambda, \mu, c_{0}, \bar{\rho}, p, s$. Due to $\left\|u_{0}\right\|_{\dot{H}^{\delta}} \leq c_{2}$, we get by (8.2) and (8.3) that

$$
\begin{aligned}
& \|u\|_{\widetilde{L}_{T}^{\infty}\left(\dot{B}_{2,2}^{-\delta}\right)} \leq C\left(1+E_{0}\right)\left(c_{2}+T\left\|\rho_{0}-\bar{\rho}\right\|_{\dot{H}^{1-\delta}}\right), \\
& \|u\|_{\widetilde{L}_{T}^{1}\left(\dot{B}_{2,2}^{2-\delta}\right)}+\|u\|_{\widetilde{L}_{T}^{2}\left(\dot{B}_{2,2}^{1-\delta}\right)} \leq C\left(c_{2}+T\left\|\rho_{0}-\bar{\rho}\right\|_{\dot{H}^{1-\delta}}\right) .
\end{aligned}
$$

Then for $r \in(1,2)$, we get by Lemma 2.1 that

$$
\begin{aligned}
& \|\nabla u\|_{L_{T}^{r}\left(L^{2}\right)} \leq \sum_{j \in \mathbf{Z}} 2^{j}\left\|\Delta_{j} u\right\|_{L_{T}^{r}\left(L^{2}\right)} \\
& \leq \sum_{j \leq 0} 2^{j} T^{\frac{1}{r}}\left\|\Delta_{j} u\right\|_{L_{T}^{\infty}\left(L^{2}\right)}+\sum_{j>0} 2^{j}\left\|\Delta_{j} u\right\|_{L_{T}^{\infty}\left(L^{2}\right)}^{1-\frac{1}{r}}\left\|\Delta_{j} u\right\|_{L_{T}^{1}\left(L^{2}\right)}^{\frac{1}{r}} \\
& \leq C\left(\|u\|_{\widetilde{L}_{T}^{\infty}\left(\dot{B}_{2,2}^{-\delta}\right)}+\|u\|_{\widetilde{L}_{T}^{1}\left(\dot{B}_{2,2}^{2-\delta}\right)}\right) \\
& \leq C\left(1+E_{0}\right)\left(c_{2}+T\left\|\rho_{0}-\bar{\rho}\right\|_{\dot{H}^{1-\delta}}\right)
\end{aligned}
$$


and similarly,

$$
\begin{aligned}
\|u\|_{L_{T}^{r}\left(L^{2}\right)} & \leq C\left(T^{\frac{1}{r}}\|u\|_{\widetilde{L}_{T}^{\infty}\left(\dot{B}_{2,2}^{-\delta}\right)}+\|\nabla u\|_{L_{T}^{r}\left(L^{2}\right)}\right) \\
& \leq C\left(1+E_{0}\right)\left(c_{2}+T\left\|\rho_{0}-\bar{\rho}\right\|_{\dot{H}^{1-\delta}}\right) .
\end{aligned}
$$

Hence, there exits a point $t_{0} \in(0, T)$ such that

$$
\left\|u\left(t_{0}\right)\right\|_{H^{1}} \leq C\left(1+E_{0}\right)\left(c_{2} / T^{\frac{2}{3}}+T^{\frac{1}{3}}\left\|\rho_{0}-\bar{\rho}\right\|_{\dot{H}^{1-\delta}}\right) .
$$

Recall that the density $\rho$ satisfies

$$
\partial_{t}(\rho-\bar{\rho})+u \cdot \nabla(\rho-\bar{\rho})=-\operatorname{div} u \rho .
$$

Making $L^{2}$ energy estimate, we get

$$
\partial_{t}\|\rho-\bar{\rho}\|_{2} \leq\|\operatorname{div} u\|_{\infty}\|\rho-\bar{\rho}\|_{2}+C\|\operatorname{div} u\|_{2} .
$$

Then by Gronwall's inequality and (8.4), we get

$$
\begin{aligned}
\|\rho(t)-\bar{\rho}\|_{2} & \leq C\left(\left\|\rho_{0}-\bar{\rho}\right\|_{2}+T^{\frac{1}{2}}\|\nabla u\|_{L_{T}^{2}\left(L^{2}\right)}\right) \\
& \leq C\left(c_{1}+T^{\frac{1}{2}}\left\|u_{0}\right\|_{2}\right) .
\end{aligned}
$$

Here we used

$$
\|\nabla u\|_{L_{T}^{2}\left(L^{2}\right)} \leq C\left(\left\|\rho_{0}-\bar{\rho}\right\|_{2}+\left\|u_{0}\right\|_{2}\right),
$$

which follows from the energy inequality (6.1). Set

$$
T_{2}=\frac{\varepsilon_{0}^{2}}{C\left(1+\left\|\rho_{0}-\bar{\rho}\right\|_{\dot{B}_{p, 1}^{\frac{3}{p}} \cap H^{s}}+\left\|u_{0}\right\|_{2}\right)^{6}},
$$

and $T_{*}=\min \left(T_{1}, T_{2}\right)$. We choose $c_{1}, c_{2}$ small enough so that

$$
C c_{1} \leq \frac{\varepsilon_{0}}{4}, \quad C\left(1+E_{0}\right) c_{2} / T_{*}^{\frac{2}{3}} \leq \frac{\varepsilon_{0}}{4},
$$

i.e, $c_{2} \leq \varepsilon_{0} T_{*}^{\frac{2}{3}} /\left(4 C\left(1+E_{0}\right)\right)$. Then it follows from (8.5) and (8.6) that

$$
\left\|a\left(t_{0}\right)\right\|_{2}+\left\|u\left(t_{0}\right)\right\|_{H^{1}} \leq \varepsilon_{0} .
$$

Theorem 6.1 ensures that

$$
\frac{c_{0}}{4} \leq \rho \leq 4 c_{0}^{-1}, \quad\|u(t)\|_{L_{T}^{\infty}\left(L^{q}\right)} \leq C .
$$

So, the solution can be extended to a global one by Theorem 7.1 ,

Acknowledgments. This work is carried out while the third author is a long term visitor at Beijing international Mathematical center of research(BIMCR). The hospitality and support of BIMCR are graciously acknowledged. Zhifei Zhang was partially supported by the NSF of China under grants 10990013 and 11071007. 


\section{References}

[1] H. Bahouri, J.-Y. Chemin and R. Danchin, Fourier analysis and nonlinear partial differential equations, Fundamental Principles of Mathematical Sciences, 343, Springer, Heidelberg, 2011.

[2] J.-M. Bony, Calcul symbolique et propagation des singularitiés pour les équations aux dérivées partielles non linéaires, Ann. de l'Ecole Norm. Sup., 14(1981), 209-246.

[3] M. Cannone, A generalization of a theorem by Kato on Naiver-Stokes equations, Revista Mat. Iber., 13 (1997), 515-541.

[4] M. Cannone, Y. Meyer and F. Planchon, Solutions autosimilaires des équations de Navier-Stokes, Séminaire "Équations aux Dérivées Partielles" de l'École polytechnique, Exposé VIII, 1993-1994.

[5] F. Charve and R. Danchin, A global existence result for the compressible Navier-Stokes equations in the critical $L^{p}$ framework, Arch. Rational Mech. Anal., 198 (2010), 233-271.

[6] J.-Y. Chemin and N. Lerner, Flot de champs de vecteurs non lipschitziens et équations de Navier-Stokes, J. Differential Equations, 121(1992), 314-328.

[7] J.-Y. Chemin and I. Gallagher, On the globa well-posedness of the 3D Navier-Stokes equations with large data, Annales de l'École Normale Supérieure, 39(2006), 679-698.

[8] J.-Y. Chemin and I. Gallagher, Large, global solutions to the Navier-Stokes equations, slowly varying in one direction, Trans. Amer. Math. Soc. , 362(2010), 2859-2873.

[9] J.-Y. Chemin, I. Gallagher and M. Paicu, Global regularity for some classes of large solutions to the Navier-Stokes equations, Ann. of Math., 173 (2011), 983-1012.

[10] J.-Y. Chemin and P. Zhang, On the global wellposedness of the 3-D incompressible anisotropic Navier-Stokes equations, Comm. Math. Phys., (272)2007, 529-566.

[11] J.-Y. Chemin, I. Gallagher and P. Zhang, Sums of large global solutions to the incompressible Navier-Stokes equations, arXiv:1002.4736v2.

[12] Q. Chen, C. Miao and Z. Zhang, Well-posedness in critical spaces for the compressible Navier-Stokes equations with density dependent viscosities, Revista Mat. Iber., 26 (2010), 915-946.

[13] Q. Chen, C. Miao and Z. Zhang, Global well-posedness for compressible Navier-Stokes equations with highly oscillating initial velocity, Comm. Pure Appl. Math.,63(2010), 1173-1224.

[14] Q. Chen, C. Miao and Z. Zhang, On the ill-posedness of the compressible Navier-Stokes equations, arXiv:1109.6092.

[15] R. Danchin, Global existence in critical spaces for compressible Navier-Stokes equations, Invent. Math., 141(2000), 579-614. 
[16] R. Danchin, Local theory in critical spaces for compressible viscous and heat-conductive gases, Comm. Partial Differential Equations, 26(2001), 1183-1233.

[17] R. Danchin, Global existence in critical spaces for flows of compressible viscous and heat-conductive gases, Arch. Rational Mech. Anal., 160(2001), 1-39.

[18] R. Danchin, On the uniqueness in critical spaces for compressible Navier-Stokes equations, Nonlinear Differential Equations Appl., 12(2005), 111-128.

[19] R. Danchin, Well-posedness in critical spaces for barotropic viscous fluids with truly not constant density, Comm. Partial Differential Equations, 32(2007), 1373-1397.

[20] E. Feireisl, A. Novotný and H. Petzeltová, On the global existence of globally defined weak solutions to the Navier-Stokes equations of isentropic compressible fluids, J. Math. Fluid Mech., 3(2001), 358-392.

[21] H. Fujita and T. Kato, On the Navier-Stokes initial value problem I, Arch. Rational Mech. Anal., 16(1964), 269-315.

[22] D. Hoff, Global solutions of the Navier-Stokes equations for multidimensional compressible flow with discontinuous initial data, J. Diff. Equa., 120(1995), 215-254.

[23] D. Hoff, Discontinuous solutions of the Navier-Stokes equations for multidimensional flows of heat-conducting fluids, Arch. Rational Mech. Anal., 139(1997), 303-354.

[24] D. Hoff, Dynamics of singularity surfaces for compressible, viscous flows in two space dimensions, Comm. Pure Appl. Math., 55(2002), 1365-1407.

[25] D. Hoff, Compressible flow in a half-space with Navier boundary condtions, J. Math. Fluid Mech., 7(2005), 315-338.

[26] X. Huang, J. Li and Z. Xin, Global well-posedness of classical solutions with large oscillations and vacuum to the three dimensional isentropic compressible Navier-Stokes equations, arXiv:1004.4749.

[27] S. Jiang and P. Zhang, Global spherically symmetric solutions of the compressible isentropic Navier-Stokes equations, Comm. Math. Phys., 215(2001), 559-581.

[28] S. Jiang and P. Zhang, Axisymmetric solutions of the 3-D Navier-Stokes equations for compressible isentropic flows, J. Math. Pure Appl., 82(2003), 949-973.

[29] P.-L. Lions, Mathematical Topics in Fluid Mechanics. Vol.2, Compressible models, Oxford University Press, 1998.

[30] A. Matsumura and T. Nishida, The initial value problem for the equations of motion of compressible viscous and heat-conductive fluids. Proc. Japan Acad. Ser. A Math. Sci. 55(1979), 337-342.

[31] J. Nash, Le problème de Cauchy pour les équations différentielles d'un fluide général, Bulletin de la Soc. Math. de France, 90(1962),487-497. 
[32] M. Paicu and Z. Zhang, Global regularity for the Navier-Stokes equations for some classes of large initial data, Analysis and PDE, 4(2011), 95-113.

[33] Y. Sun, C. Wang and Z. Zhang, A Beale-Kato-Majda blow-up criterion for the 3-D compressible Navier-Stokes equations, J. Math. Pure Appl., 95(2011), 36-47.

[34] Z. Xin, Blowup of smooth solutions to the compressible Navier-Stokes equation with compact density, Comm. Pure Appl. Math., 51(1998), 229-240.

[35] T. Zhang, Global solutions of compressible Navier-Stokes equations with a densitydependent viscosity coefficient, arXiv:0904:1659.

[36] T. Zhang, Global wellposed problem for the 3-D incompressible anisotropic Navier-Stokes equations in an anisotropic space, Comm. Math. Phys., 287(2009), 211-224. 